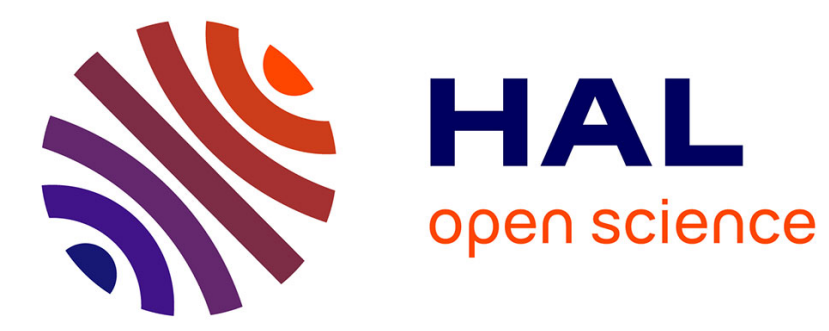

\title{
HYPERBOLICITY NOTIONS FOR VARIETIES DEFINED OVER A NON-ARCHIMEDEAN FIELD
}

Rita Rodríguez Vázquez

\section{To cite this version:}

Rita Rodríguez Vázquez. HYPERBOLICITY NOTIONS FOR VARIETIES DEFINED OVER A NON-ARCHIMEDEAN FIELD. 2017. hal-01672776

\section{HAL Id: hal-01672776 \\ https://hal.inria.fr/hal-01672776}

Preprint submitted on 27 Dec 2017

HAL is a multi-disciplinary open access archive for the deposit and dissemination of scientific research documents, whether they are published or not. The documents may come from teaching and research institutions in France or abroad, or from public or private research centers.
L'archive ouverte pluridisciplinaire HAL, est destinée au dépôt et à la diffusion de documents scientifiques de niveau recherche, publiés ou non, émanant des établissements d'enseignement et de recherche français ou étrangers, des laboratoires publics ou privés. 


\title{
HYPERBOLICITY NOTIONS FOR VARIETIES DEFINED OVER A NON-ARCHIMEDEAN FIELD
}

\author{
RITA RODRÍGUEZ VÁZQUEZ
}

\begin{abstract}
Firstly, we pursue the work of W. Cherry on the analogue of the Kobayashi semi distance $d_{\mathrm{CK}}$ that he introduced for analytic spaces defined over a non-Archimedean metrized field $k$. We prove various characterizations of smooth projective varieties for which $d_{\mathrm{CK}}$ is an actual distance.

Secondly, we explore several notions of hyperbolicity for a smooth algebraic curve $X$ defined over $k$. We prove a non-Archimedean analogue of the equivalence between having negative Euler characteristic and the normality of certain families of analytic maps taking values in $X$.
\end{abstract}

\section{Contents}

1. Introduction

2. Berkovich analytic spaces 6

\begin{tabular}{lll}
\hline 3. Smooth analytic curves & 9
\end{tabular}

\begin{tabular}{lr}
\hline 4. Cherry hyperbolicity & 16
\end{tabular}

5. Zalcman's reparametrization lemma 18

6. Further notions of hyperbolicity 22

7. Curves with non-negative Euler characteristic 28

\begin{tabular}{lll}
\hline 8. Analytic maps on special domains & 29
\end{tabular}

9. Proof of Theorem|D 33

\begin{tabular}{|ll}
\hline $10 . \quad$ Proof of Theorem|C & 35
\end{tabular}

$\begin{array}{ll}\text { References } & 37\end{array}$

\section{INTRODUCTION}

The notion of Kobayashi hyperbolicity Kob67 is arguably one of the fundamental notions in complex geometry. We refer to [Kob98] and Lan87] for detailed monographics on the topic or to the more recent surveys [Voi03, DR11.

It is a fundamental and remarkable fact that hyperbolic compact complex spaces can be characterized as follows:

Date: December 26, 2017.

Research supported by the ERC grant Nonarcomp no. 307856. 
Theorem 1. Let $X$ be a smooth compact complex analytic space endowed with a hermitian metric. The following conditions are equivalent:

(1) The space $X$ is Kobayashi hyperbolic.

(2) The derivative of any holomorphic map from the unit disk $\mathbb{D}$ to $X$ is bounded on every compact subset $\mathrm{K}$ of $\mathbb{D}$ by a constant depending only on $X$ and $\mathrm{K}$.

(3) The space $X$ contains no entire curve.

(4) The family $\operatorname{Hol}(\mathbb{D}, X)$ is normal.

For instance, a compact Riemann surface is hyperbolic if and only if its genus is at least two, and no abelian variety is hyperbolic.

Recall that a family of holomorphic maps $f_{n}: \mathbb{D} \rightarrow X$ is normal if it is equicontinuous, and by Ascoli-Arzelà's theorem this means that up to extracting a subsequence, the sequence $f_{n}$ converges uniformly on every compact subset to a holomorphic map $g: \mathbb{D} \rightarrow X$. The main content of the above theorem is the implication $(3) \Rightarrow(1)$, known as Brody's lemma [Bro78].

Complex analytic spaces that are hyperbolic enjoy remarkable properties concering the compactness of the spaces of holomorphic maps with values in them. De Franchis' theorem, generalized by Sam66, KO75, Nog92, asserts that there exist only finitely many meromorphic surjective maps from a compact variety into a compact hyperbolic variety.

We now fix a a non-Archimedean complete valued field $k$ that is nontrivially valued and algebraically closed. In this paper, we explore analogues of the previous theorem for analytic spaces defined over $k$. We shall work in the context of analytic spaces as developped by V. Berkovich in [Ber90, Ber93. Being locally compact and locally pathwise connected, such spaces have good topological properties, what makes them an adapted framework to arguments of analytic nature.

In Che96, the author translates the definition of Kobayashi chains on complex spaces [Kob67] to the set of rigid points $X(k)$ of a Berkovich space $X$, which gives rise to the Cherry-Kobayashi semi distance $d_{\mathrm{CK}}$ on $X(k)$. We will say that an analytic space $\mathrm{X}$ is Cherry hyperbolic if $d_{\mathrm{CK}}$ is an actual distance. This semi distance shares several properties with its complex counterpart. On the unit disk, $d_{\mathrm{CK}}$ agrees with the standard distance, and $d_{\mathrm{CK}}$ is contracting for analytic maps.

In a series of papers Che93, Che96, ACW08, Che94, Cherry studied in detail the behaviour of $d_{\mathrm{CK}}$ and the existence of entire curves in the case of abelian varieties and of projective curves, making extensive use of the reduction theory available for these varieties. His results contrast with the complex case. Indeed, any abelian variety $X$ is Cherry hyperbolic and contains no entire curve, i.e. every analytic map $\mathbb{A}^{1, \text { an }} \rightarrow X$ is constant.

In the non-Archimedean setting, limits of analytic maps need not be analytic so that the notion of normality has to be slightly modified, see [FKT12]. 
Roughly speaking, a family of analytic maps from a (boundaryless) analytic space $X$ to a compact space $Y$ is normal whenever every sequence admits a subsequence that is pointwise converging to a continuous map. In RV16] we proved a version of Montel's theorem in this setting for families of analytic maps with values in an affinoid space.

Inspired by a remarkable conjecture by Cherry formulated in Che94, Che96 it is natural to ask whether the following holds.

Conjecture 1. Let $X$ be a smooth compact boundaryless $k$-analytic space. The following conditions are equivalent:

(1) The space $X$ is Cherry-Kobayashi hyperbolic.

(2) The space $X$ contains no entire curve.

(3) The space $X$ contains no rational curve.

(4) The family $\operatorname{Mor}_{k}(\mathbb{D}, X)$ of analytic maps from $\mathbb{D}$ to $X$ is normal.

Cherry treated the equivalence between (1), (2) and (3) in the cases of curves, abelian varieties and certain large classes of compact algebraic surfaces in Che93, §VII.3]. We continue the study of the relations between the previous properties under the assumption that the field $k$ has zero residue characteristic. This hypothesis is necessary to control the size of the image of a disk under an analytic map in terms of the norm of its derivative.

We restrict our attention to smooth projective varieties, for which there is a natural way to measure the norm of a derivative. Let $X$ be a smooth projective variety defined over $k$. We fix a projective embedding of $X \subset \mathbb{P}_{k}^{N}$, and consider the restriction of the spherical distance $d_{\mathbb{P}}$ to $X$, which allows us to define the Fubini-Study derivative $\left|f^{\prime}(z)\right|$ of any analytic map $f: \mathbb{D} \rightarrow X$.

To simplify notations, we set $d_{\mathrm{CK}}^{\prime}:=\min \left\{1, d_{\mathrm{CK}}\right\}$. Our first result reads as follows:

Theorem A. Let $X$ be a smooth projective variety defined over an algebraically closed non-Archimedean complete field $k$ of residue characteristic zero. The following conditions are equivalent:

i) Every rigid point $x \in X$ has a neighbourhood $U$ such that the semi distances $d_{\mathrm{CK}}^{\prime}$ and $d_{\mathbb{P}}$ are equivalent on $U(k)$.

ii) The semi distance $d_{\mathrm{CK}}$ defines the same topology as $d_{\mathbb{P}}$ on rigid points.

iii) For every rigid point in the open unit disk $\mathbb{D}$ there exists a neighbourhood $U$ and a positive constant $C$ such that

$$
\sup _{f \in \operatorname{Mor}_{k}(\mathbb{D}, X)} \sup _{z \in U}\left|f^{\prime}(z)\right| \leq C .
$$

The main content of this statement resides in the implication ii) $\Rightarrow$ iii), which results from an adaptation of Zalcman's lemma Zal75 to the nonArchimedean setting. After renormalizing, we obtain a sequence of analytic maps $g_{n}: \mathbb{D}(0 ; n) \rightarrow X$ whose Fubini-Study derivative is uniformly bounded 
on every compact subset of $\mathbb{A}^{1 \text {,an }}$ and does not vanish at at 0 . A major difference with the complex case is that this sequence does not converge a priori to an entire curve $\mathbb{A}^{1, \text { an }} \rightarrow X$.

Notice that in the complex case, the assertion ii) is satisfied if and only if $X$ is Kobayashi hyperbolic by a theorem of Barth [Lan87, I, §2].

The hypothesis on the residue characteristic of $k$ is only used for the implication iii) $\Rightarrow$ i).

Observe that condition iii) in Theorem A implies that the family $\operatorname{Mor}_{k}(\mathbb{D}, X)$ of analytic maps from the open unit disk into $X$ is normal at every rigid point. The converse implication seems likely to hold. Conjecture 1 shows that if the family $\operatorname{Mor}_{k}(\mathbb{D}, X)$ is normal at every rigid point, then $X$ is Cherry hyperbolic, which is slightly weaker than condition ii).

Our next result answers affirmatively to Conjecture 1 in the case of smooth projective curves over a field $k$ of zero residue characteristic.

Theorem B. Let $X$ be a smooth projective curve defined over an algebraically closed field of residue characteristic zero. The following conditions are equivalent:

i) The curve $X$ has positive genus.

ii) Every rigid point $x \in X$ has a neighbourhood $U$ such that the semi distances $d_{\mathrm{CK}}^{\prime}$ and $d_{\mathbb{P}}$ are equivalent on $U(k)$.

iii) The Fubini-Study derivative of $\operatorname{Mor}_{k}(\mathbb{D}, X)$ is uniformly bounded in a neighbourhood of every rigid point.

iv) The family $\operatorname{Mor}_{k}(\mathbb{D}, X)$ is normal.

v) The curve $X$ is Cherry hyperbolic.

Observe that Theorem A implies the equivalence between ii) and iii) and the implication ii) $\Rightarrow \mathrm{v}$ ). For the other implications we rely on the semistable reduction theorem, which implies that the image of a disk under an analytic map is again an analytic disk (except if $X$ is the projective line).

The equivalence between i) and v) without any hypothesis on the characteristic of $k$ was proved in Che96 by embedding a projective curve into its Jacobian.

The previous theorems are not fully satisfactory, since abelian varieties satisfy all the equivalent conditions above, but carry many self-maps and thus violate De Franchis' theorem and its generalizations. It would be particularly interesting to characterize those varieties $X$ for which similar compactness properties hold for any family of analytic maps with values in $X$. Our next result addresses this problem in the case of smooth algebraic curves.

Recall any smooth (irreducible) algebraic curve $X$ can be uniquely embedded into a smooth projective curve $\bar{X}$ such that $\bar{X} \backslash X$ is a finite set of $k$-points. The Euler characteristic of $X$ is then defined by

$$
\chi(X)=2-2 g-\#(\bar{X} \backslash X),
$$


where $g$ denotes the genus of $\bar{X}$. Complex algebraic curves with negative Euler characteristic are precisely those that are Kobayashi hyperbolic, and for which any family of holomorphic maps $Y \rightarrow X$ is normal for every analytic space $Y$.

Theorem C. Suppose that $k$ is a complete non-Archimedean algebraically closed field of zero residue characteristic whose residue field is countable. Let $X$ be a smooth irreducible algebraic curve over $k$.

Then, the Euler characteristic $\chi(X)$ of $X$ is negative if and only if any sequence of analytic maps $f_{n}: U \rightarrow X$ admits a subsequence $f_{n_{j}}$ that converges pointwise to a continuous map $f_{\infty}: U \rightarrow \bar{X}$ such that either $f_{\infty}(U) \subset X$ or $f_{\infty}$ is constant equal to a point in $\bar{X} \backslash X$.

Recall that $\chi(X) \leq 0$ if and only if $\operatorname{Mor}_{k}(\mathbb{D}, X)$ is normal, the projective case being a consequence of Theorem $B$.

One implication was already noticed in [FKT12]. When the Euler characteristic of $X$ is non-negative, then we may find a smooth boundaryless curve $U$ such that the family of all analytic maps $\operatorname{Mor}_{k}(U, X)$ is not normal. When $X$ is the projective or the affine line, one can take $U$ to be the unit disk. When $X$ is the punctured affine line any open annulus works. In \$7, we extend these arguments to any elliptic curve, in which case we may take $U=X$.

The core of the proof lies in the forward implication: the family $\operatorname{Mor}_{k}(U, X)$ is normal as soon as $\chi(X)<0$. Recall that the skeleton of a curve consists of the points that do not have a neighbourhood isomorphic to an open disk. We consider first the case where the skeleton of $X$ is not too small and next the general case.

Theorem D. Suppose that $k$ is a complete non-Archimedean algebraically closed field of zero residue characteristic. Let $X$ be a smooth irreducible algebraic curve of negative Euler characteristic whose skeleton $\mathrm{S}^{\mathrm{an}}(X)$ is not a singleton.

Let $U$ be a smooth connected boundaryless analytic curve. Then there exists a finite affinoid cover $\left(\bar{X}_{i}\right)$ of $\bar{X}$ and a locally finite cover $\left(U_{j}\right)$ of $U$ by basic tubes such that for every analytic map $f: U \rightarrow X$ and every $j$ the image $f\left(U_{j}\right)$ is contained in some affinoid $\bar{X}_{i}$.

Moreover, the affinoid cover $\left(\bar{X}_{i}\right)$ is independent of $U$.

Notice that this theorem shows a form of equicontinuity for maps from $U$ to $X$. This result together with Montel's theorem [RV16] implies a stronger form of Theorem $\mathrm{C}$ when $\mathrm{S}^{\mathrm{an}}(X)$ is not a singleton.

When the skeleton of $X$ is reduced to a point, then $X$ is a projective curve and admits a smooth model over the valuation ring $k^{\circ}$. In other words, it is a curve with good reduction. In this case, our arguments use in a crucial manner the hypothesis that the residue field $\tilde{k}$ is countable. 
This paper is structured as follows. In 2 we review some basic facts on Berkovich spaces and in $\$ 3$ on smooth analytic curves. The CherryKobayashi semi distance is introduced and discussed in 84 . Our non-Archimedean version of Zalcman's lemma is proved in \$5. Theorems $\mathrm{A}$ and $\mathrm{B}$ are proved in 86 . Section 8 contains several preparatory results needed for Theorems D and C, which are proved in $\$ 9$ and $\$ 10$ respectively.

Acknoledgements. I would like to thank Jérôme Poineau and William Cherry for their valuable comments on an earlier version of this paper.

This research was supported by the ERC grant Nonarcomp no. 307856.

\section{Berkovich anAlytic SPACES}

In this section, we review some aspects of Berkovich analytic spaces. We fix once and for all a non-Archimedean non-trivially valued field $k$ that is algebraically closed. We refer to [Ber90, Ber93, Tem15] for a thorough discussion of this theory.

2.1. Good analytic spaces. Given a positive integer $N$ and an $N$-tuple of positive real numbers $r=\left(r_{1}, \cdots, r_{N}\right)$, we denote by $k\left\{r^{-1} T\right\}$ the set of power series $f=\sum_{I} a_{I} T^{I}, I=\left(i_{1}, \cdots, i_{N}\right)$, with coefficients $a_{I} \in k$ such that $\left|a_{I}\right| r^{I} \rightarrow 0$ as $|I|:=i_{1}+\cdots+i_{N}$ tends to infinity. The norm $\left\|\sum_{I} a_{I} T^{I}\right\|=\max _{I}\left|a_{I}\right| r^{I}$ makes $k\left\{r^{-1} T\right\}$ into a Banach $k$-algebra. When $r=(1, \cdots, 1)$, this algebra is called the Tate algebra and we denote it by $\mathcal{T}_{n}$

A Banach $k$-algebra $\mathcal{A}$ is called affinoid if there exists an admissible surjective morphism of $k$-algebras $\varphi: k\left\{r^{-1} T\right\} \rightarrow \mathcal{A}$. If $r_{i} \in\left|k^{\times}\right|$for all $i$, then $\mathcal{A}$ is said to be strictly affinoid. It is a fundamental fact that all $k$-affinoid algebras are noetherian and that all their ideals are closed, see [Ber90, Proposition 2.1.3]. Notice that the fact that the epimorphism $\varphi$ is admissible implies that $\mathcal{A}$ and $k\left\{r^{-1} T\right\} / \operatorname{ker}(\varphi)$ endowed with the residue norm are isomorphic as Banach algebras.

The analytic spectrum $\mathcal{M}(\mathcal{A})$ of a Banach $k$-algebra $(\mathcal{A},\|\|$.$) is the set$ of all mutiplicative seminorms on $\mathcal{A}$ that are bounded by the norm $\|$.$\| on$ $\mathcal{A}$. Given $f \in \mathcal{A}$, its image under a seminorm $x \in \mathcal{M}(\mathcal{A})$ is denoted by $|f(x)| \in \mathbb{R}_{+}$. The set $\mathcal{M}(\mathcal{A})$ is endowed with the weakest topology such that all the functions of the form $x \mapsto|f(x)|$ with $f \in \mathcal{A}$ are continuous. The resulting topological space is nonempty, compact and Hausdorff [Ber90, Theorem 1.2.1].

Given a point $x \in \mathcal{M}(\mathcal{A})$, the fraction field of $\mathcal{A} / \operatorname{Ker}(x)$ naturally inherits from $x$ an absolute value extending the one on $k$. Its completion is the complete residue field at $x$ and denoted by $\mathcal{H}(x)$.

The analytic spectrum $X=\mathcal{M}(\mathcal{A})$ of a $k$-affinoid algebra $\mathcal{A}$ is called a $k$-affinoid space. When $\mathcal{A}$ is strictly affinoid, one says that $X$ is strictly affinoid. 
The affinoid space $X$ naturally carries a sheaf of analytic functions $\mathcal{O}_{X}$, see [Ber90, §2.3].

Example 2.1. The closed polydisk of dimension $N$ and polyradius $r=$ $\left(r_{1}, \cdots, r_{N}\right) \in\left(\mathbb{R}_{*}^{+}\right)^{N}$ is defined to be $\overline{\mathbb{D}}^{N}(r):=\mathcal{M}\left(k\left\{r^{-1} T\right\}\right)$. The Gauss point $x_{g} \in \overline{\mathbb{D}}^{N}$ is the point associated to the norm

$$
\left|\left(\sum a_{I} T^{I}\right)\left(x_{g}\right)\right|:=\max \left|a_{I}\right| \text {. }
$$

When $r=(1, \cdots, 1)$ we just write $\overline{\mathbb{D}}^{N}$, and when $N=1$ we denote it by $\overline{\mathbb{D}}$.

Example 2.2. Pick any real numbers $r \leq R$. The closed annulus is the affinoid space $A[r, R]:=\mathcal{M}\left(k\left\{R^{-1} T, r S\right\} /(S T-1)\right)$. It can be identified with the closed subset of the closed disk $\overline{\mathbb{D}}(R)$ consisting of the points $x \in \overline{\mathbb{D}}(R)$ with $r \leq|T(x)| \leq R$.

In the following, we shall exclusively work with the category of good analytic spaces which is formed by the subcategory of analytic spaces (defined in [Ber90]) that are locally ringed spaces modelled on affinoid spaces. In other words, any point in a good analytic space admits a neighbourhood isomorphic to an affinoid space.

Example 2.3. The open polydisk of dimension $N$ and polyradius $r \in\left(\mathbb{R}_{*}^{+}\right)^{N}$ is the set

$$
\mathbb{D}_{k}^{N}(r)=\left\{x \in \overline{\mathbb{D}}^{N}(r):\left|T_{i}(x)\right|<r_{i}, i=1, \ldots, N\right\} .
$$

It can be naturally endowed with a structure of good analytic space by writing it as the increasing union of $N$-dimensional polydisks $\overline{\mathbb{D}}_{k}^{N}(\rho)$ whose radii $\rho=\left(\rho_{1}, \cdots, \rho_{N}\right) \in\left(\left|k^{\times}\right|\right)^{N}$ satisfy $\rho_{i}<r_{i}$ for all $i=1, \ldots, N$.

Example 2.4. Pick any real numbers $r<R$. The open annulus is the set

$$
A(r, R)=\{x \in A[r, R]: r<|T(x)|<R\} .
$$

It can be naturally endowed with a structure of good analytic space by writing it as an increasing union of closed annuli.

2.2. Analytification of algebraic varieties. A fundamental class of good analytic spaces are the analytifications of algebraic varieties. To every algebraic variety $X$ over $k$ one can associate a $k$-analytic space $X^{\text {an }}$ in a functorial way. We refer to [Ber90, §3.4] for a detailed construction.

In the case of an affine variety $X=\operatorname{Spec}(A)$, where $A$ is a finitely generated $k$-algebra, then the set $X^{\text {an }}$ consists of all the multiplicative seminorms on $A$ whose restriction to $k$ coincides with the norm on $k$. This set is endowed with the weakest topology such that all the maps of the form $x \in X^{\text {an }} \mapsto|f(x)|$ with $f \in A$ are continuous. Fix an embedding of $X$ into some affine space $\mathbb{A}^{N}$. Then the intersections with the open polydisks $X^{\text {an }} \cap \mathbb{D}^{N}(r)$ with $r>0$ define an analytic atlas on $X^{\text {an }}$.

Observe that any $k$-point $x \in X$ corresponds to a morphism of $k$-algebras $A \rightarrow k$ and its composition with the norm on $k$ defines a rigid point in $X^{\text {an }}$. 
Since $k$ is algebraically closed, one obtains in this way an identification of the set of closed points in $X$ with the set of rigid points in $X^{\text {an }}$.

Let $X$ be a general algebraic variety and fix an affine open cover. The analytification of a general algebraic variety $X$ is obtained by glueing together the analytification of its affine charts in natural way. Analytifications of algebraic varieties are good analytic spaces, and closed points are in natural bijection with rigid points as in the affine case.

We refer to the next section for a description of the topology of the analytification of an algebraic curve.

2.3. Basic tubes. Special analytic spaces will play an important role in the proof of Theorem $B$. We thus introduce the following terminology.

Definition 2.5. A $k$-analytic space $X$ is called a basic tube if there exists a reduced equidimensional strictly $k$-affinoid space $\hat{X}$ and a closed point $\tilde{x}$ in its reduction such that $X$ is isomorphic to $\operatorname{red}^{-1}(\tilde{x})$.

By convention, a basic tube is reduced.

Theorem 2.6. A basic tube is connected.

The fact that any basic tube over an algebraically closed field is connected is a deep theorem due to [Bos77], which was generalized to arbitrary base fields in Poi14.

Example 2.7. Let $a_{1}, \cdots, a_{m}$ be type II points in $\mathbb{P}^{1, \text { an }}$. Then every connected component of $\mathbb{P}^{1, \text { an }} \backslash\left\{a_{1}, \cdots, a_{m}\right\}$ is a basic tube.

Analytic spaces come with a natural notion of boundary and interior. We refer to [Ber90, §3.1] for the definitions for good $k$-analytic spaces and to [Ber93, §1.5.4] for a discussion in the case of general Berkovich spaces.

Recall that a topological space is $\sigma$-compact if it is the union of countably many compact subspaces. For instance, open Berkovich polydisks or the analytification of an algebraic variety are $\sigma$-compact spaces. Observe that there exist simple examples of $k$-analytic spaces which are not $\sigma$-compact, e.g. the closed unit disk of dimension $N \geq 2$ with the Gauss point removed over a base field $k$ with uncountable reduction $\tilde{k}$. The following propositions, whose proofs can be found in [], imply that every basic tube is boundaryless and $\sigma$-compact.

Proposition 2.8. A k-analytic space $X$ is a basic tube if and only if it is isomorphic to a connected component of the interior of some equidimensional strictly $k$-affinoid space.

Proposition 2.9. For every basic tube $X$ there exist a strictly $k$-affinoid space $\hat{X}$ and a distinguished closed immersion into some closed polydisk $\hat{X} \rightarrow$ $\overline{\mathbb{D}}^{N}$ such that $X$ is isomorphic to $\hat{X} \cap \mathbb{D}^{N}$.

Remark 2.10. Every good reduced boundaryless $k$-analytic space has a basis of open neighbourhooods that are basic tubes. 
2.4. Normal families. Recall the definition of normal family from [FKT12].

Definition 2.11. Let $U$ be a boundaryless $k$-analytic space and $X$ a compact $k$-analytic space. A family of analytic maps $\mathcal{F}$ from $U$ to $X$ is normal at a point $z \in U$ if there exists a neighbourhood $V \ni z$ where every sequence $\left\{f_{n}\right\}$ in $\mathcal{F}$ admits a subsequence $\left\{f_{n_{j}}\right\}$ converging pointwise to a continuous map. The family $\mathcal{F}$ is normal if it is normal at every point $z \in U$.

We shall use the following result from [RV16]:

Theorem 2.12. Let $k$ be a non-Archimedean complete field that is non trivially valued and $X$ a good, reduced, $\sigma$-compact, boundaryless strictly $k$ analytic space. Let $Y$ be a strictly $k$-affinoid space.

Then, every sequence of analytic maps $f_{n}: X \rightarrow Y$ admits a pointwise converging subsequence whose limit is continuous.

\section{Smooth Analytic CURVES}

In this section, we recall some facts on the structure of smooth analytic curves. Our main references are [Ber90, §4] and [Duc14].

3.1. The analytic affine line. Recall that the analytic affine line $\mathbb{A}^{1 \text {, an }}$ is the set of bounded seminorms on the polynomial ring $k[T]$. The points in $\mathbb{A}^{1, \text { an }}$ can be explicitly described as follows [Ber90, §1.4.4].

Pick $a \in k$ and $r \in \mathbb{R}_{+}$and denote by $\bar{B}(a ; r)$ the closed ball in $k$ centered at $a$ and of radius $r$. To $\bar{B}(a ; r)$ one can associate a point $\eta_{a, r} \in \mathbb{A}^{1 \text {,an }}$ by setting $\left|P\left(\eta_{a, r}\right)\right|:=\sup _{|y-a| \leq r}|P(y)|$ for every polynomial $P \in k[T]$. If $r=0$, then $\eta_{a, 0}$ corresponds to evaluating polynomials in $a \in k$.

More generally, any decreasing sequence of closed balls $\bar{B}\left(a_{i} ; r_{i}\right)$ in $k$ defines a sequence of points $\eta_{a_{i}, r_{i}}$ that converges in $\mathbb{A}^{1 \text {,an }}$ to a point $\eta \in \mathbb{A}^{1 \text {,an }}$ sending any polynomial $P \in k[T]$ to $|P(\eta)|=\lim _{i}\left|P\left(\eta_{a_{i}, r_{i}}\right)\right|$. Observe that such a sequence of balls might have empty intersection, in which case $\lim _{i} r_{i}=r>0$ since $k$ is complete.

It is a key fact due to Berkovich [Ber90, $\S 1.4 .4]$ that any point in $\mathbb{A}^{1 \text {,an }}$ comes from a decreasing sequence of closed balls in $k$.

Suppose that $x=\lim \eta_{a_{i}, r_{i}}$ and set $\bar{B}=\cap_{i} \bar{B}\left(a_{i} ; r_{i}\right)$. V. Berkovich introduced the following terminology.

i) The point $x$ is of type I if and only if $\bar{B}=\{a\}$, with $a \in k$.

ii) The point $x$ is of type II if and only if $\bar{B}=\bar{B}(a ; r)$ with $r \in\left|k^{\times}\right|$.

iii) The point $x$ is ot type III if and only if $\bar{B}=\bar{B}(a ; r)$ with $r \notin\left|k^{\times}\right|$.

iv) The point $x$ is of type IV if and only if $\bar{B}=\emptyset$.

Every point in $\mathbb{A}^{1 \text {,an }}$ falls into one of these four types.

The analytic projective line $\mathbb{P}^{1, \text { an }}$ is the one-point compactification of $\mathbb{A}^{1, \text { an }}$. An open (resp. closed) disk in $\mathbb{P}^{1, \text { an }}$ is either an open (resp. closed) 
disk in $\mathbb{A}^{1 \text {,an }}$ or the complement of a closed (resp. open) disk in $\mathbb{A}^{1, \text { an }}$. Connected affinoid domains in $\mathbb{P}^{1 \text {,an }}$ are the complement of finitely many open disks.

3.2. First properties of analytic curves. We extend the description of the previous section to arbitrary curves. Recall that a $k$-analytic curve $X$ is a $k$-analytic space that is Hausdorff and of pure dimension 1 . Throughout this section, $X$ will denote a smooth analytic curve over $k$.

Points in a $k$-analytic curve can be classified as follows, see [Duc14, §3.3]. Let $x \in X$ and let $\mathcal{H}(x)$ be its complete residue field. Then one says that:

i) The point $x$ is of type I if $\mathcal{H}(x) \simeq k$;

ii) The point $x$ is of type II if the reduction $\widetilde{\mathcal{H}(x)}$ has transcendence degree 1 over $\tilde{k}$ and $|\mathcal{H}(x)|=|k|$;

iii) The point $x$ is of type III if $\widetilde{\mathcal{H}(x)} \simeq \tilde{k}$ and the value group $\left|\mathcal{H}(x)^{\times}\right|$ is generated by $\left|k^{\times}\right|$and some real number $r \notin\left|k^{\times}\right|$;

iv) The point $x$ is of type IV if $\widetilde{\mathcal{H}(x)} \simeq \tilde{k},|\mathcal{H}(x)|=|k|$ and $\mathcal{H}(x)$ is a non-trivial extension of $k$.

On $\mathbb{A}^{1 \text {,an }}$, this classification of the points in an analytic curve agrees with the one introduced above.

Let $x$ be a type II point in a $k$-analytic curve $X$. We define the genus $g(x)$ of the point $x$ as the genus of the unique smooth projective curve $C$ over $\tilde{k}$ whose field of rational functions is isomorphic to $\widetilde{\mathcal{H}(x)}$. The set of points in $X$ with positive genus is a closed discrete subset of $X$ by [Duc14, Théorème 4.4.17].

The following fundamental topological result will be used in the sequel, see [Duc14, Théorème 4.5.10]:

Theorem 3.1. Every $k$-analytic curve is paracompact.

3.3. Graph structure. Following the terminology of [Duc14, we say that a locally compact Hausdorff topological space $X$ is a graph if it admits a fundamental basis of open sets $U$ satisfying the following properties:

i) For every pair of points $x, y \in U$, there exists a unique closed subset $[x, y] \subset U$ homeomorphic to a segment of endpoints $x$ and $y$.

ii) The boundary of $U$ in $X$ is finite.

A graph $X$ is a tree if $X$ itself satisfies property i).

It follows from the definition that graphs are locally path-connected and that trees are path-connected. And a topological space $X$ is a graph if and only if it is Hausdorff and every point has a neighbourhood that is a tree [Duc14, 1.3.3.1].

It is a fundamental fact that every $k$-analytic curve is a graph Duc14, Théorème 3.5.1]. 
Let $X$ be a $k$-analytic curve. Given a point $x \in X$, the tangent space $T_{x} X$ at $x$ is defined as the set of connected components of $U \backslash\{x\}$ where $U$ is an open neighborhood of $x$ which is a tree. It can be also defined as the set of paths leaving from $x$ modulo the relation having a common initial segment proving that the definition does not depend on the choice of $U$. Given any tangent direction $\vec{v} \in T_{x} X$, we denote by $U(\vec{v})$ the open subset of points $y \in X \backslash\{x\}$ such that there exists a path starting from $y$ and abuting at $x$ in the direction of $\vec{v}$.

3.4. Skeleton of an analytic curve. Recall the definition of the skeleton of an analytic curve:

Definition 3.2. The skeleton of a curve $X$ is the set of all points $x \in X$ having no neighbourhood isomorphic to an open disk. It will be denoted by $\mathrm{S}^{\mathrm{an}}(X)$.

The only smooth projective curve with empty skeleton is $\mathbb{P}^{1 \text {,an }}$, see Duc14, $\S 5.4 .8]$. In the non-compact case, examples of curves with empty skeleton include the open disk and the affine line. The skeleton of an open annulus $A(\rho, 1)$ is the segment consisting of the points $\eta_{0, r}$ for $\rho<r<1$.

By definition, $\mathrm{S}^{\text {an }}(X)$ is a closed subset of $X$ and its complement is a disjoint union of open disks. The skeleton of $X$ is a closed locally finite subgraph of $X$. If $X$ is projective, then $\mathrm{S}^{\text {an }}(X)$ is compact.

For any curve $X$ with nonempty skeleton there is a retraction map $r_{X}$ : $X \rightarrow \mathrm{S}^{\text {an }}(X)$, defined as follows. Every point in the skeleton is fixed by $r_{X}$. For every point $x \in X \backslash S^{\text {an }}(X)$, denote by $U_{x}$ the maximal open neighbourhood of $x$ that is isomorphic to $\mathbb{D}$. Then, $r_{X}(x)$ is the unique point in the topological boundary of $U_{x}$ in $X$. The retraction map is continuous.

Let us now introduce the notion of nodes of a curve $X$ following [Duc14, Lemme 6.2.3], which is a subset of its skeleton. In order to define it, recall that we say that a tangent direction $\vec{v} \in T_{x} X$ at a type II or type III point $x \in X$ is discal if $U(\vec{v})$ is a disc.

Definition 3.3. Let $X$ be a smooth analytic curve over $k$. A type II point $x \in \mathrm{S}^{\mathrm{an}}(X)$ is a node if one of the following conditions is satisfied:

i) The point $x$ has positive genus;

ii) There exist three distinct tangent directions at $x$ that are non-discal;

iii) The point $x$ belongs to the boundary of $X$.

The set of all nodes of a curve $X$ will be denoted by $\mathrm{N}(X)$. Observe that it contains every branching point in $S^{\text {an }}(X)$, which is discrete and closed as $\mathrm{S}^{\mathrm{an}}(X)$ is a locally finite graph. Since the boundary of $X$ is finite and the set of points $x \in X$ of positive genus is closed and discrete Duc14, Théorème 4.4.7], then $\mathrm{N}(X)$ is also discrete and closed. The complement of $\mathrm{N}(X)$ in $\mathrm{S}^{\mathrm{an}}(X)$ is by definition a disjoint union of open segments. It follows that if 
$\mathrm{N}(X)$ is nonempty, then $X \backslash \mathrm{N}(X)$ is a disjoint union of infinitely many open disks, finitely many open annuli, and finitely many punctured disks.

3.5. Smooth projective curves. Let us describe in more detail the structure of smooth projective curves. Recall that in this case, the skeleton is compact and the set of nodes finite.

Let $X$ be a smooth irreducible projective curve. Its genus $g$ is encoded in the topology of the skeleton and in the points of positive genus as follows, see [Duc14, §5.2.6] and [Ber90, §4.3]. Denote by $b$ the first Betti number of $\mathrm{S}^{\mathrm{an}}(X)$. Then, one has the equality

$$
g=b+\sum_{x \in X_{[2]}} g(x)
$$

where $X_{[2]}$ is the set of type II points in $X$. Notice that the sum is finite, since a smooth projective curve $X$ has only finitely many points of positive genus.

We remark that the endpoints of the skeleton are nodes:

Lemma 3.4. Let $X$ be a smooth projective curve. Let $\eta$ be an endpoint of $\mathrm{S}^{\mathrm{an}}(X)$. Then $\eta$ is a node and has positive genus.

This result helps us describe all the possibilities for a smooth projective curve $X$ in terms of its skeleton and its nodes, see [Duc14, §5.4.12].

i) If $X$ has empty skeleton, then it is isomorphic to $\mathbb{P}^{1, \text { an }}$.

ii) If $\mathrm{S}^{\mathrm{an}}(X)$ is nonempty and $X$ has no nodes, then $\mathrm{S}^{\mathrm{an}}(X)$ has no endpoints by Lemma 3.4, and hence the skeleton must be homeomorphic to a circle. It follows from (3.1) that $X$ has genus 1 . In that case, we say that $X$ is a Tate curve, i.e. the analytification of an elliptic curve whose $j$-invariant is not integral.

iii) If $\mathrm{N}(X)$ is nonempty, then it follows from (3.1) that $X$ has positive genus.

The particular case where $X$ has only one node $\eta_{X}$ deserves a more thorough description. We distinguish two possibilities for the geometry of $X$ based on its skeleton, which will be used in the proof of Theorem $\mathrm{C}$ and $\mathrm{D}$.

i) The skeleton of $X$ consists only of the point $\eta_{X}$. In that case, we say that $X$ has good reduction. By (3.1), the genus of $\eta_{X}$ equals the genus of the curve $X$. In particular, if $g\left(\eta_{X}\right)=1$ then the curve $X$ is the analytification of an elliptic curve with bad reduction, i.e. whose $j$-invariant is integral.

ii) There is at least one loop in $\mathrm{S}^{\mathrm{an}}(X)$ passing through $\eta_{X}$. By (3.1), $X$ has genus at least 2 .

Proof of Lemma 3.4. Let $\eta$ be an endpoint of the skeleton of $X$. If $\mathrm{S}^{\mathrm{an}}(X)=$ $\{\eta\}$, then $\eta$ is a node, since otherwise it has an open neighbourhood that is isomorphic to an open disk. As $X$ is boundaryless and $\mathrm{S}^{\text {an }}(X)$ has no branching points, we conclude that $\eta$ has positive genus. 
We may assume that $\{\eta\}$ is strictly contained in $\mathrm{S}^{\mathrm{an}}(X)$. As $\eta \in \mathrm{S}^{\mathrm{an}}(X)$, there exists a tangent direction $\vec{v} \in T_{\eta} X$ such that $U(\vec{v}) \cap S^{\text {an }}(X)$ is nonempty, and so $\vec{v}$ is non-discal. Being an endpoint of $\mathrm{S}^{\mathrm{an}}(X)$, all the other tangent directions at $\eta$ are discal. Since $X$ is projective, the only remaining possibility for $\eta$ to be a node is to have positive genus, since $\eta$ is not a branching point of the skeleton.

Suppose by contradiction that $g(\eta)=0$. By [Duc14, Théorème 4.5.4], then point $\eta$ has a simply connected open neighbourhood $V$. Moreover, this theorem states that $V$ is isomorphic to an open annulus if $\eta$ is a type III point. If $\eta$ is a type II point, the same result implies that we may reduce $V$ such that it is also isomorphic to an open annulus, since $\eta$ is an endpoint of $\mathrm{S}^{\mathrm{an}}(X)$.

In fact, as every tangent direction $\vec{v}^{\prime} \in T_{\eta} X$ different from $\vec{v}$ is discal, we may assume that every $U\left(\vec{v}^{\prime}\right)$ is contained in $V$. After maybe reducing $V$, we may assume that he topological boundary of $V$ in $X$ is a single point in $\mathrm{S}^{\text {an }}(X)$ and that no point in $V$ has positive genus. By Duc14, Proposition 5.1.18], $V$ is isomorphic to an open disk, contradicting the fact that $\eta \in$ $\operatorname{S}^{\mathrm{an}}(X)$.

We have the following description of curves of positive genus:

Lemma 3.5. Let $X$ be a smooth irreducible projective curve with non-empty set of nodes. Then $\mathrm{S}^{\mathrm{an}}(X)$ can be decomposed as the disjoint union of $\mathrm{N}(X)$ and open segments $I_{1}, \ldots, I_{a}$, with each $I_{j}$ isomorphic to a real segment $\left(1, R_{j}\right)$ with $R_{j} \in\left|k^{\times}\right|$, for $1 \leq j \leq a$. The complement of $\mathrm{N}(X)$ in $X$ is a disjoint union of infinitely many open unit disks and annuli $A\left(1, R_{1}\right), \ldots, A\left(1, R_{a}\right)$.

Moreover, if $\mathrm{N}(X)$ consists of a single node $\eta_{X}$, then the closure $\bar{I}_{j}=$ $I_{j} \cup\left\{\eta_{X}\right\}$ of each $I_{j}$ in $X$ is a circle.

Proof. Let $X$ be a smooth projective curve with non-empty set of nodes. Suppose that $\mathrm{S}^{\mathrm{an}}(X) \backslash \mathrm{N}(X)$ contains a loop $C$. As the skeleton of $X$ is connected and every branching point in $\mathrm{S}^{\mathrm{an}}(X)$ is a node, we conclude that $C=\mathrm{S}^{\text {an }}(X)$, contradicting the fact that $\mathrm{N}(X)$ is nonempty.

Moreover, since $X$ is projective there are only finitely many nodes, which are all type II points. As a consequence, the set $S^{\text {an }}(X) \backslash \mathrm{N}(X)$ consists of finitely many open segments $I_{j}$ isomorphic to real segments $\left(1, R_{j}\right)$ with $R_{j} \in\left|k^{\times}\right|$, for $1 \leq j \leq a$. It follows that $X \backslash \mathrm{N}(X)$ consists of a disjoint union of open disks and the open annuli $A\left(1, R_{1}\right), \ldots, A\left(1, R_{a}\right)$.

Assume now that $X$ has only one node $\eta_{X}$. If $S^{\text {an }}(X)$ consists only of the point $\eta_{X}$, then the complement of $N(X)$ is a disjoint union of open disks. Otherwise, the exists at least one loop in $\mathrm{S}^{\text {an }}(X)$ passing through $\eta_{X}$ by Lemma 3.4. Necessarily, the closure of each segment $I_{j}$ in $X$ is $I_{j} \cup\left\{\eta_{X}\right\}$, which is homeomorphic to a circle.

The following lemma will be essential in the subsequent chapters, specially the proof of Theorem C. It is a particular case of [Duc14, Proposition 6.1.2]. 
Lemma 3.6. Let $X$ be a smooth irreducible projective curve over $k$ and $x \in X$ a rigid point. Every open neigbourhood $U$ of $x$ has an open subset $V \subseteq U$ such that $X \backslash V$ is an affinoid domain of $X$.

3.6. Geometry of basic tubes of dimension 1. A one-dimensional basic tube has empty skeleton if and only if it is isomorphic to an open disk, see [Duc14, Proposition 5.1.18]. An open annulus $A(R, 1)$ with $R \in\left|k^{\times}\right|$is a basic tube, and its skeleton is isomorphic to the open real segment $(R, 1)$.

A point $x$ in the skeleton of a smooth analytic curve $X$ is a node if it satisfies one of the following conditions: the point $x$ is a branching point of the skeleton, $x$ has positive genus or it belongs to the boundary of $X$.

An important class of basic tubes of dimension one are star-shaped domains:

Definition 3.7. A basic tube $U$ of dimension 1 is called a star-shaped domain if it is simply connected and contains exactly one node $\eta_{U}$.

Let us describe the geometry of star-shaped domains in more detail. Let $U$ be a star-shaped domain. Since basic tubes are boundaryless, the point $\eta_{U}$ has positive genus or it is a branching point of $S^{\text {an }}(U)$. The skeleton of $U$ can be decomposed in a disjoint union of $\left\{\eta_{U}\right\}$ and finitely many open segments. Thus, every connected component of $U \backslash\left\{\eta_{U}\right\}$ is either isomorphic to an open disk or to an open annulus. The latter correspond to the non-discal tangent directions in $T_{\eta_{U}} U$.

As a consequence, a star-shaped domain $U$ determines the following data:

i) The residue curve at $\eta_{U}$, which is the unique smooth projective curve $C_{U}$ over $\tilde{k}$ such that $\tilde{k}\left(C_{U}\right) \simeq \widetilde{\mathcal{H}\left(\eta_{U}\right)}$. The curve $C_{U}$ has genus $g(U)$;

ii) A reduced divisor $D_{U}$ on $C_{U}$ whose support is the set of non-discal directions at $\eta_{U}$;

iii) For every non-discal direction $\vec{v} \in T_{\eta_{U}} U$ a real number $\rho \in\left|k^{\times}\right|$of norm less than 1 such that the open set $U(\vec{v})$ is isomorphic to the open annulus $A(\rho, 1)$.

3.7. Analytic maps between curves. The following result will be systematically used in the sequel, see [Duc14, Lemme 6.2.4]:

Lemma 3.8. Let $X$ be a smooth projective curve over $k$ and $U$ a basic tube of dimension 1 .

Let $f: U \rightarrow X$ a non-constant analytic map. Let $z \in U$ be a type II or III point and consider the tangent map $d f(z): T_{z} U \rightarrow T_{f(z)} X$. If a tangent direction $\vec{v} \in T_{z} U$ is discal, then so is $d f(z)(\vec{v})$.

As an immediate consequence, we have:

Lemma 3.9. Let $X$ be a smooth projective curve and $f: \mathbb{D} \rightarrow X$ an analytic map. Then the image of $f$ is contained in some connected component of $X \backslash \mathrm{S}^{\mathrm{an}}(X)$. 
Another important fact that will be used throughout the present chapter is the following proposition:

Proposition 3.10. Let $U$ be a basic tube of dimension 1 that is not analytically isomorphic to the unit disk. Let $X$ be a smooth projective curve and $f: U \rightarrow X$ an analytic map. If a point $z \in \mathrm{S}^{\mathrm{an}}(U) \backslash \mathrm{N}(U)$ is such that $f(z)$ lies in the skeleton of $X$, then the connected component of $S^{\text {an }}(U) \backslash \mathrm{N}(U)$ containing $z$ is mapped to $\mathrm{S}^{\mathrm{an}}(X)$.

The proof relies on the following Lemma [Duc14, Lemme 6.2.5]:

Lemma 3.11. Let $X$ be a smooth projective curve over $k$ and $U$ a basic tube of dimension 1.

Let $f: U \rightarrow X$ a non-constant analytic map. If $f(z)$ is a node in $\mathrm{S}^{\mathrm{an}}(X)$, then $z$ is a node in $\mathrm{S}^{\mathrm{an}}(U)$.

Proof of Proposition 3.10. Let $z \in \mathrm{S}^{\mathrm{an}}(U)$ be a non-nodal point such that $f(z) \in \mathrm{S}^{\mathrm{an}}(X)$. By Lemma 3.11, the point $f(z)$ is not a node. In particular, $f(z)$ cannot be an endpoint of the skeleton by Lemma 3.4. and so both $z$ and $f(z)$ have exactly two non-discal tangent directions.

Consider the complement of $\mathrm{N}(U)$ in $\mathrm{S}^{\text {an }}(U)$, and let $I$ be the connected component containing the point $z$. Suppose by contradiction that not the whole $I$ is mapped to the skeleton of $X$. In this case, we may find a point $z^{\prime} \in I$ such that $f\left(z^{\prime}\right) \in \mathrm{S}^{\text {an }}(X)$ and such that a non-discal direction $\vec{v} \in T_{z^{\prime}} U$ is mapped to some discal direction at $f\left(z^{\prime}\right)$. The tangent map $d f\left(z^{\prime}\right): T_{z^{\prime}} U \rightarrow$ $T_{f\left(z^{\prime}\right)} X$ is surjective, and so there is a discal direction at $z^{\prime}$ that is mapped to a non-discal direction at $f\left(z^{\prime}\right)$. This contradicts Lemma 3.8 .

We shall use the following version of Hurwitz's theorem during the proof of Theorem C:

Proposition 3.12. Let $U$ be a boundaryless connected curve over $k$ and $X$ a $k$-affinoid space. Let $Z$ be any closed analytic subset of $X$.

Suppose that $f_{n}$ is a sequence of analytic maps from $U$ to $X \backslash Z$ converging pointwise to a continuous map $g$.

Then, we have either that $g(U) \cap Z=\emptyset$ or $g(U) \subset Z$.

Proof. The set $Z$ is the zero locus of some analytic function $\varphi$ in the affinoid algebra of $X$ with $|\varphi|_{\text {sup }} \leq 1$. Since the zeros of $\varphi$ form a finite subset of $X$ of rigid points, we may assume that $X$ is the closed unit disk and that $Z$ is the origin by replacing $f_{n}$ by $\varphi \circ f_{n}$.

In this case our assumption ensures that the functions $h_{n}=\log \left|f_{n}\right|: X \rightarrow$ $\mathbb{R}_{-}$are harmonic. It follows from [Thu05, Proposition 3.1.2] that either $h_{n}$ converges uniformly to $-\infty$ on compact subsets and so $g(U) \subset\{0\}$, or any limit map of the sequence $h_{n}$ is still harmonic, in which case one necessarily has $g(U) \cap\{0\}=\emptyset$. 


\section{Cherry hyperbolicity}

4.1. Cherry's notion of hyperbolicity. Recall the definition of the CherryKobayashi semi distance [Che96]:

Definition 4.1. Let $x, y$ be rigid points in a $k$-analytic space $X$. A Kobayashi chain joining $x$ and $y$ is a finite set of analytic maps $f_{l}: \overline{\mathbb{D}} \rightarrow X$ and points $z_{l}, w_{l} \in \overline{\mathbb{D}}(k), l=1, \cdots, m$ such that $f_{1}\left(z_{1}\right)=x, f_{l}\left(w_{l}\right)=f_{l+1}\left(z_{l+1}\right)$ for $l=1, \cdots, m-1$ and $f_{m}\left(w_{m}\right)=y$. The Cherry-Kobayashi semi distance on $X$ is defined by

$$
d_{\mathrm{CK}}(x, y)=\inf \sum_{l=1}^{m}\left|w_{l}-z_{l}\right|,
$$

where the infimum is taken over all Kobayashi chains joining $x$ and $y$. If there is no Kobayashi chain joining $x$ and $y$, we set $d_{\mathrm{CK}}(x, y)=\infty$.

Observe that the group of analytic automorphisms of $\overline{\mathbb{D}}$ is the set of series of the form $\sum_{n>0} a_{n} T^{n}$ such that $\left|a_{1}\right|=1$ and such that $\max _{n}\left|a_{n}\right| \leq 1$, which are isometries for the distance |.|. Thus, $d_{\mathrm{CK}}$ is invariant under automorphisms of $\overline{\mathbb{D}}$. Up to composition by such an automorphism we may suppose that $z_{l}=0$, for all $l$.

On the closed disk $\overline{\mathbb{D}}$, the Cherry-Kobayashi semi distance agrees with the distance induced by the norm on $k$.

Remark 4.2. Not every point pair of points in an analytic space $X$ can be joined by a Kobayashi chain. Assume for instance that $X$ has dimension at least two and take a point $x \in X$ such that the transcendance degree of $\widetilde{\mathcal{H}(x)}$ over $\tilde{k}$ greater than one. Then, no analytic map $\mathbb{D} \rightarrow X$ avoids the point $x$.

Definition 4.3. A k-analytic space $X$ is Cherry hyperbolic if $d_{\mathrm{CK}}$ is an actual distance on $X(k)$, which might take the value $\infty$.

As $\mathbb{A}^{1, \text { an }}$ can be written as a union of disks of whose radii tend to infinity, we see that $d_{\mathrm{CK}}$ is exactly zero on $\mathbb{A}^{1 \text {,an }}$, just like over $\mathbb{C}$.

The semi distance $d_{\mathrm{CK}}$ shares an important property with its complex counterpart: analytic maps are distance decreasing with respect to $d_{\mathrm{CK}}$. As

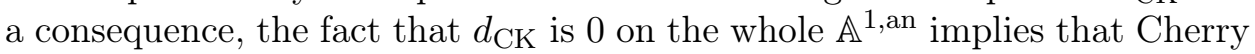
hyperbolicity is stronger than Brody hyperbolicity, i.e. the non-existence of entire curves.

Recall from $\$ 3$ that the skeleton $S^{\text {an }}(X)$ of a curve $X$ is the set of points not having a neighbourhood that is isomorphic to an open disk. Let $X$ be an elliptic curve and pick any two distinct rigid points $x, y \in X$. If $x$ and $y$ belong to the same connected component of $X \backslash \mathrm{S}^{\mathrm{an}}(X)$, then $d_{\mathrm{CK}}(x, y)$ agrees with the distance on the disk, and hence $d_{\mathrm{CK}}(x, y) \neq 0$. Otherwise, they cannot be joined by a Kobayashi chain and thus $d_{\mathrm{CK}}(x, y)=\infty$. Thus, elliptic curves are Cherry hyperbolic. The same argument shows that a projective curve $X$ is Cherry-hyperbolic if and only if it has strictly positive genus. 
We refer to [Che96] for further details on the Cherry-Kobayashi semi distance.

4.2. Alternate definition of the Kobayashi semi distance. Let us briefly comment on the following alternate definition of an analogue of the Kobayashi semi distance on the set of rigid points of a $k$-analytic space $X$, which is very natural. In the same notation as above, for any $x, y \in X(k)$ we set

$$
d(x, y)=\inf \max _{1 \leq l \leq m}\left|w_{l}-z_{l}\right|,
$$

where the infimum is taken over all Kobayashi chains joining $x$ and $y$. As before, if there is no Kobayashi chain joining $x$ and $y$ we set $d_{\mathrm{CK}}(x, y)=\infty$. The obtained semi distance $d$ satisfies the ultrametric inequality.

Remark 4.4. Let $X$ be a smooth analytic curve, and pick any two rigid points $x, y$. Observe that there exists a Kobayashi chain joining $x$ and $y$ if and only if they belong to the same connected component of $X \backslash \mathrm{S}^{\mathrm{an}}(X)$. In this case, the chain consists of a single analytic map $f: \overline{\mathbb{D}} \rightarrow X$, and as a consequence we have that $d=d_{\mathrm{CK}}$.

Clearly, $d \leq d_{\mathrm{CK}}$ for any analytic space $X(k)$. In general, these semidistances are not equivalent, as shown in the following example.

Indeed, pick a closed unit disk and take two rigid points $x, y \in \overline{\mathbb{D}}$ with $|x-y|=1$. Attach to $\overline{\mathbb{D}}$ two irreducible components $X_{1}$ and $Y$ isomorphic to $\overline{\mathbb{D}}$, one passing through $x$ and the other through $y$. For every integer $n \geq 3$, take rigid points $x_{1}^{(n)} \in X_{1}, y_{n} \in Y$ such that $\left|x-x_{1}^{(n)}\right|=\left|y-y_{n}\right|=\frac{1}{n}$. Attach a closed disk to $X_{1}$ passing through $x_{1}^{(n)}$. Denote this new irreducible component by $X_{2}^{(n)}$ and pick a rigid point $x_{2}^{(n)} \in X_{2}^{(n)}$ with $\left|x_{1}^{(n)}-x_{2}^{(n)}\right|=\frac{1}{n}$. Repeat this procedure as to obtain irreducible components $X_{l}^{(n)} \simeq \overline{\mathbb{D}}$ and rigid points $x_{l}^{(n)} \in X_{l}^{(n)}$ for $1 \leq l \leq n$ with $X_{n}^{(n)}=Y, x_{n-1}^{(n)}=y_{n}$ and $x_{n}^{(n)}=y$. Denote by $x_{0}^{(n)}:=x$ for every $n \geq 3$. Observe that $\left|x_{l}^{(n)}-x_{l-1}^{(n)}\right|=\frac{1}{n}$ for every $l=1, \ldots, n$.

For every $n$, the points $x=x_{0}^{(n)}, x_{1}^{(n)}, \ldots, x_{n}^{(n)}=y$ form a Kobayashi chain joining $x$ and $y$. We see that

$$
d(x, y)=\inf _{n \geq 3}\left|x_{1}^{(n)}-x_{0}^{(n)}\right|=\inf _{n \geq 3} \frac{1}{n}=0,
$$

whereas

$$
d_{\mathrm{CK}}(x, y)=\inf _{n \geq 3} \sum_{l=1}^{n}\left|x_{l}^{(n)}-x_{l-1}^{(n)}\right|=\inf _{n \geq 3} n \cdot \frac{1}{n}=1 .
$$

In the sequel, we shall build on W. Cherry's work and only consider the semi distance $d_{\mathrm{CK}}$. 
4.3. Royden's length function. Royden's length function on the tangent bundle of a complex manifold has the particularity that the semi distance it defines on the manifold is precisely the Kobayashi semi distance. This enables us to translate the notion of Kobayashi hyperbolicity into infinitesimal terms. We refer to Roy71 for further details.

One can adapt this definition to Berkovich spaces as follows. Recall that the tangent space of a smooth analytic space $X$ at a point $x$ is the set of all derivations on the local ring $\mathcal{O}_{X, x}$.

Definition 4.5. Let $X$ be a smooth analytic space over $k$. For every $x \in$ $X(k)$, Royden's length function is defined for every $\vec{v} \in T_{x} X$ as

$$
|\vec{v}|_{\text {Roy }}:=\inf \left\{\frac{1}{|\lambda|}: \exists f: \mathbb{D} \rightarrow X \text { analytic, } f(0)=x, f^{\prime}(0)=\lambda \vec{v}\right\} .
$$

The following result holds for fields $k$ of arbitrary characteristic:

Proposition 4.6. Let $X$ be a smooth projective variety over some nonArchimedean field $k$.

If Royden's function is such that $|\vec{v}|_{\text {Roy }}=0$ if and only if $\vec{v}=0$, then $X$ contains no entire curve.

Proof. Suppose there exists an entire curve $f: \mathbb{A}^{1, \text { an }} \rightarrow X$. We may suppose that $f$ is not constant at 0 , and hence $\vec{v}=f^{\prime}(0) \in T_{f(0)} X$ is a non-zero vector. Denoting by $m_{n}: \mathbb{D} \rightarrow \mathbb{D}(0 ; n)$ the homothety of ratio $n$, the sequence $f_{n}:=f \circ m_{n}: \mathbb{D} \rightarrow X$ is such that $f_{n}^{\prime}(0)=\lambda_{n} \vec{v}$, with $\left|\lambda_{n}\right|=n$. Hence, $|\vec{v}|_{\text {Roy }}=0$.

Proposition 4.7. Let $X$ be a smooth projective variety defined over a complete non-Archimedean field $k$ of characteristic zero.

Assume that every rigid point admits a neighbourhood on which the FubiniStudy derivative of $\operatorname{Mor}_{k}(\mathbb{D}, X)$ is uniformly bounded. Then, Royden's function is such that $|\vec{v}|_{\text {Roy }}=0$ if and only if $\vec{v}=0$.

Proof. Suppose that there exists a point $x \in X$ and some nonzero $\vec{v} \in T_{x} X$ such that $|\vec{v}|_{\text {Roy }}=0$. Then there is a sequence $f_{n}: \mathbb{D} \rightarrow X$ fixing the origin and such that $f_{n}^{\prime}(0)=R_{n} v$, with $v \neq 0$ and $\left|R_{n}\right| \rightarrow+\infty$. Hence, the Fubini-Study derivative explodes at 0 .

\section{ZALCMAN'S REPARAMETRIZATION LEMMA}

5.1. Fubini-Study derivative. We fix once and for all homogeneous coordinates on $\mathbb{P}^{N \text {,an }}$ and $\mathbb{P}^{1, \text { an }}$.

Definition 5.1. Let $\Omega$ be any open subset of $\mathbb{A}^{1 \text {,an }}$. Consider an analytic map $f: \Omega \rightarrow \mathbb{P}^{N \text {,an }}$ and choose coordinates $f=\left[f_{0}: \cdots: f_{N}\right]$. The FubiniStudy derivative of $f$ at a point $z \in \Omega$, is

$$
\left|f^{\prime}(z)\right|=\max \left\{1,|z|^{2}\right\} \frac{\max \left|\left(f_{i}^{\prime} f_{j}-f_{j}^{\prime} f_{i}\right)(z)\right|}{\max \left|f_{i}(z)\right|^{2}} .
$$


Observe that, by construction, the function $z \in \Omega \mapsto\left|f^{\prime}(z)\right|$ is continuous. Next we prove some properties of the Fubini-Study derivative.

Lemma 5.2. For every analytic map $f: \mathbb{D} \rightarrow \mathbb{P}^{N \text {,an }}$ given in homogeneous coordinates by $f=\left[f_{0}: \cdots: f_{N}\right]$, we have

$$
\left|f^{\prime}(z)\right| \leq \frac{\max \left\{\left|f_{i}^{\prime}(z)\right|\right\}}{\max \left\{\left|f_{i}(z)\right|\right\}}
$$

The proof is trivial.

Since rigid points are dense, for any analytic map $f: \Omega \rightarrow \mathbb{P}^{N \text {,an }}$ we have:

$$
\sup _{z \in \Omega}\left|f^{\prime}(z)\right|=\sup _{z \in \Omega(k)}\left|f^{\prime}(z)\right| \text {. }
$$

A direct computation shows:

Lemma 5.3. Let $\Omega, \Omega^{\prime}$ be open subsets of $\mathbb{A}^{1, \text { an }}$. Consider analytic maps $g: \Omega \rightarrow \mathbb{P}^{1 \text {,an }}$, and $f: \Omega^{\prime} \rightarrow \mathbb{P}^{N \text {,an }}$ with $g(\Omega) \subseteq \Omega^{\prime}$. Then, we have

$$
\left|(f \circ g)^{\prime}(z)\right|=\left|f^{\prime}(g(z))\right| \cdot\left|g^{\prime}(z)\right| .
$$

Corollary 5.4. For every $g \in \operatorname{PGL}\left(2, k^{\circ}\right)$ and every analytic map $f: \Omega \rightarrow$ $\mathbb{P}_{k}^{1, \text { an }}$, we have $\left|(f \circ g)^{\prime}(z)\right|=\left|f^{\prime}(z)\right|$.

Proof. We have to prove that $\left|g^{\prime}(z)\right|=1$ for every $g \in \operatorname{PGL}\left(2, k^{\circ}\right)$. A simple calculation shows that it holds for every $g$ of the form $z \mapsto a z, z \mapsto z+b$ and $z \mapsto 1 / z$, with $|a|=1$ and $|b| \leq 1$. Since $\operatorname{PGL}\left(2, k^{\circ}\right)$ is generated by all the maps of this form, the assertion is proved.

5.2. Diameter function. It will be useful in the sequel to estimate the size of the image of a disk. The required tool is the diameter function.

There are diameter functions on $\mathbb{A}^{1 \text {,an }}$ and on $\mathbb{P}^{1, \text { an }}$. Recall from $\$ 3.1$ that any point $x \in \mathbb{A}^{1 \text {,an }}$ is uniquely determined by a decreasing sequence of disks $\left\{\bar{B}\left(a_{i} ; r_{i}\right)\right\}$ in $k$. The diameter function is defined as $\operatorname{diam}_{\mathbb{A}}(x)=\lim r_{i}$. On $\mathbb{P}^{1, \text { an }}$, one sets

$$
\operatorname{diam}(x)=\frac{\operatorname{diam}_{\mathbb{A}}(x)}{\max \left\{1,|x|^{2}\right\}} .
$$

In both cases, a point has zero diameter if and only if it is rigid.

Observe that $\operatorname{diam}_{\mathbb{A}}(x)=\inf _{c \in k}|(T-c)(x)|$. We refer to [BR10, §2.7] for further details on the diameter function on $\mathbb{P}^{1, \text { an }}$.

We now extend these definitions to any dimension.

Definition 5.5. For any $x \in \mathbb{A}^{N \text {,an }}$, we set:

$$
\operatorname{diam}_{\mathbb{A}}(x):=\max _{1 \leq i \leq N} \inf _{c_{i} \in k}\left|\left(T_{i}-c_{i}\right)(x)\right|=\max _{1 \leq i \leq N} \operatorname{diam}_{\mathbb{A}} \pi_{i}(x),
$$

where $\pi_{i}: \mathbb{A}^{N \text {,an }} \rightarrow \mathbb{A}^{1 \text {,an }}$ is the usual projection to the $i$-th coordinate.

Definition 5.6. Let $x \in \mathbb{P}^{N \text {,an }}$. Choose an affine chart isomorphic to $\mathbb{A}^{N \text {,an }}$ at $x$. We may assume $x=\left[1: x_{1}: \cdots: x_{N}\right]$. Write $\left|x_{i}\right|=\left|T_{i}(x)\right|$. We set:

$$
\operatorname{diam}(x):=\frac{\operatorname{diam}_{\mathbb{A}}(x)}{\max \left\{1,\left|x_{i}\right|^{2}\right\}}=\frac{\max _{1 \leq i \leq N} \operatorname{diam}_{\mathbb{A}} \pi_{i}(x)}{\max \left\{1,\left|x_{i}\right|^{2}\right\}} .
$$


It is clear from the definitions that $\operatorname{diam}_{\mathbb{A}}$ and diam are zero exactly on the rigid points.

Lemma 5.7. The function diam $: \mathbb{P}^{N, \text { an }} \rightarrow \mathbb{R}_{\geq 0}$ is invariant under the action of $\operatorname{PGL}\left(N+1, k^{\circ}\right)$.

Proof. The function diam is clearly invariant under translations of the form $T_{i} \mapsto T_{i}-a_{i}, a_{i} \in k^{\circ}$.

It follows directly from the definition that for $a_{1}, \ldots, a_{N} \in k$ with $\left|a_{i}\right|=1$,

$$
\operatorname{diam}\left(x_{1}, \cdots, x_{N}\right)=\operatorname{diam}\left(a_{1} x_{1}, \cdots, a_{N} x_{N}\right) .
$$

Finally, consider maps of the form

$$
\varphi:\left[1: x_{1}: \cdots: x_{N}\right] \mapsto\left[x_{i}: x_{1}: \cdots: x_{i-1}: 1: x_{i+1}: \cdots: x_{N}\right] .
$$

Clearly, we have $\operatorname{diam}(\varphi(x))=\operatorname{diam}(x)$. All these transformations generate $\operatorname{PGL}\left(N+1, k^{\circ}\right)$.

Lemma 5.8. Assume that $\operatorname{char}(\tilde{k})=0$, and consider an analytic map $f$ : $\mathbb{D} \rightarrow \mathbb{P}^{N \text {,an }}$. Then for every $z \in \mathbb{D}$, we have

$$
\operatorname{diam}(f(z)) \leq \operatorname{diam}(z) \cdot\left|f^{\prime}(z)\right| .
$$

Moreover, for $N=1$ we have an equality.

Remark 5.9. The previous lemma does not hold if $\operatorname{char}(\tilde{k})=p>0$. In fact, there are maps with small Fubini-Study derivative and whose image is arbitrarily big. Take for instance the sequence $f_{n}: z \mapsto c_{n} z^{p^{n}}$, with $\left|c_{n}\right|=$ $\left(p^{n}\right)^{p^{n}}$. Denote by $\eta_{0, \epsilon}$ the point in $\mathbb{D}$ associated to the closed ball $\bar{B}(0 ; \epsilon)$. A direct computation shows that

It follows that

$$
\left|f_{n}^{\prime}\left(\eta_{0, \epsilon}\right)\right|=\frac{\left|c_{n}\right| \epsilon^{p^{n}-1}}{p^{n} \max \left\{1,\left|c_{n}\right| \epsilon^{p^{n}}\right\}^{2}} .
$$

$$
\sup _{n} \sup _{z \in \mathbb{D}}\left|f_{n}^{\prime}(z)\right|=1
$$

If $\epsilon<p^{-n}$, then the Fubini-Study derivative is $\left|f_{n}^{\prime}\left(\eta_{0, \epsilon}\right)\right|=\left(p^{n} \epsilon\right)^{p^{n}-1}$. Thus, we see that $\operatorname{diam}\left(f_{n}\left(\eta_{0, \epsilon}\right)\right)$ cannot be bounded away from 1 uniformly in $\epsilon$ and $n \in \mathbb{N}$.

Proof of Lemma 5.8. Let us first consider the case $N=1$. By continuity, it suffices to consider points of type II and III. More so, we may assume that $z=\eta_{0, r} \in \mathbb{D}$ (i.e. $z$ is associated to the closed ball $\bar{B}(0 ; r) \subset k$ ) and that $f(z)=\eta_{0, R}$, since both the diameter function and the Fubini-Study derivative are invariant under the action of $\operatorname{PGL}\left(2, k^{\circ}\right)$ by Lemmas 5.4 and 5.7 .

Let $f(z)=\sum_{i \geq 0} a_{i} z^{i}$ be the series development of $f$. It follows from the definitions that the equality is equivalent to $\operatorname{diam}_{\mathbb{A}}\left(f\left(\eta_{0, r}\right)\right)=\operatorname{diam}\left(\eta_{0, r}\right)$. $\left|f^{\prime}\left(\eta_{0, r}\right)\right|$. We have

$$
\operatorname{diam}_{\mathbb{A}}\left(f\left(\eta_{0, r}\right)\right)=\max _{i \geq 1}\left|a_{i}\right| r^{i}=r \cdot \max _{i \geq 1}\left|a_{i}\right| \cdot|i| r^{i-1}=r \cdot\left|f^{\prime}\left(\eta_{0, r}\right)\right|,
$$


concluding the proof for $N=1$.

Consider now the general case. We may choose homogeneous coordinates $\left[z_{0}, \cdots, z_{N}\right]$ in $\mathbb{P}^{N \text {,an }}$ such that the inverse image under $f$ of the hyperplane $H_{\infty}=\left\{z_{0}=0\right\}$ is a discrete subset of $\mathbb{D}$. Denote by $\Gamma$ its convex hull in $\mathbb{D}$. It suffices to prove the result for points $z \in \mathbb{D}$ lying outside $\Gamma$. On a neighbourhood $U$ of $z$ contained in $\mathbb{D} \backslash \Gamma$, the map $f$ can be expressed as a $\operatorname{map} f: U \rightarrow \mathbb{A}^{N, \text { an }}$, i.e. $f=\left[1: f_{1}, \cdots: f_{N}\right]$.

By the previous case, we know that

$$
\begin{aligned}
\operatorname{diam}_{\mathbb{A}}(f(z)) & =\max _{1 \leq i \leq n} \operatorname{diam}(z) \cdot\left|\left(\pi_{i} \circ f\right)^{\prime}(z)\right| \\
& =\operatorname{diam}(z) \max _{1 \leq i \leq n}\left|f_{i}^{\prime}(z)\right|
\end{aligned}
$$

By Lemma 5.2, we see that

$$
\operatorname{diam}(f(z))=\operatorname{diam}(z) \frac{\max _{1 \leq i \leq n}\left|f_{i}^{\prime}(z)\right|}{\max \left\{1,\left|f_{i}(z)\right|^{2}\right\}} \leq \operatorname{diam}(z) \cdot\left|f^{\prime}(z)\right|,
$$

proving the assertion.

5.3. Zalcman's reparametrization lemma. We follow the proof found in [Ber06]. Notice that our result does not imply that the reparametrized sequence is converging.

Proposition 5.10. Let $X$ be a smooth projective variety defined over an algebraically closed complete non-Archimedean field $k$.

Suppose that there exists a sequence of analytic maps $f_{n}: \mathbb{D} \rightarrow X$ whose Fubini-Study derivative is not locally uniformly bounded in a neighbourhood of some rigid point $z_{0} \in \mathbb{D}$. Then, we can find a sequence of rigid points $z_{n} \rightarrow z_{0}$ and a sequence $k \ni \rho_{n} \rightarrow 0$ such that the rescaled sequence $g_{n}(z):=$ $f_{n}\left(z_{n}+\rho_{n} z\right)$ satisfies the following properties:

i) Each $g_{n}$ is defined on the open disk of radius $n$;

ii) The Fubini-Study derivatives of the maps $g_{n}$ are uniformly bounded on any compact subset of $\mathbb{A}_{k}^{1 \text {,an }}$;

iii) For every $n \in \mathbb{N}$ we have $\left|g_{n}^{\prime}(0)\right|=1$.

The proof relies on the following technical result, whose proof we transpose directly to the non-Archimedean setting.

Lemma 5.11 (Gromov). Let $\varphi: \overline{\mathbb{D}}(0 ; R) \rightarrow \mathbb{R}_{+}$be a locally bounded function, and fix $\epsilon>0$ and $\tau>1$. Then, for every $a \in \overline{\mathbb{D}}(0 ; R)(k)$ such that $\varphi(a)>0$, there is $b \in \overline{\mathbb{D}}(0 ; R)(k)$ satisfying:

i) $|a-b| \leq \frac{\tau}{\epsilon(\tau-1) \varphi(a)}$

ii) $\varphi(b) \geq \varphi(a)$

iii) If $x \in \overline{\mathbb{D}}(0 ; R)(k)$ is such that $|x-b| \leq \frac{1}{\epsilon \varphi(b)}$, then $\varphi(x) \leq \tau \varphi(b)$.

Proof. Suppose we can find a point $a \in \overline{\mathbb{D}}(0 ; R)(k)$ such that every $b \in$ $\overline{\mathbb{D}}(0 ; R)(k)$ fails to satisfy one of the three conditions. In particular, so does a. As $a$ itself obviously satisfies i) and ii), there must exist a rigid point $a_{1}$ 
such that $\left|a_{1}-a\right| \leq \frac{1}{\epsilon \varphi(a)}$ and $\varphi\left(a_{1}\right)>\tau \varphi(a)$. We will show by induction that we can construct a Cauchy sequence of rigid points along which $\varphi$ is not bounded.

Suppose that we have constructed $a_{1}, \cdots, a_{n} \in \overline{\mathbb{D}}(0 ; R)(k)$ satisfying $\mid a_{i}-$ $a \mid \leq \frac{1}{\epsilon \varphi(a)}$ and $\varphi\left(a_{i}\right)>\tau^{i} \varphi(a)$. In particular, $a_{n}$ satisfies i) and ii) and hence not iii). We then find $a_{n+1}$ satisfying $\left|a_{n+1}-a_{n}\right| \leq \frac{1}{\tau^{n} \epsilon \varphi(a)}$ and $\varphi\left(a_{n+1}\right)>\tau \varphi\left(a_{n}\right)>\tau^{n+1} \varphi(a)$. The ultrametric inequality now shows that $\left|a_{n+1}-a\right| \leq \frac{1}{\epsilon \varphi(a)}$ and that $\left|a_{n+j}-a_{n}\right| \leq \frac{1}{\tau^{n} \epsilon \varphi(a)}$ for every positive integer $j$. Thus, $\left\{a_{n}\right\}$ is a Cauchy sequence and must converge to some rigid point $\alpha$, but we have shown that $\varphi$ is not bounded at $\alpha$.

Proof of Proposition 5.10. We may suppose $z_{0}=0$, and $X=\mathbb{P}_{k}^{N \text {,an }}$.

Pick a sequence of rigid points $a_{n} \rightarrow 0$ such that $\left|f_{n}^{\prime}\left(a_{n}\right)\right| \geq n^{3}$. For every $a_{n}$, we now apply Lemma 5.11 chosing $\epsilon=1 / n, \tau_{n}=1+\frac{1}{n}$ and $\varphi=\left|f_{n}^{\prime}\right|$ and obtain a sequence $z_{n} \in \mathbb{D}(k)$ satisfying:

i) $\left|a_{n}-z_{n}\right| \leq \frac{n^{2}+n}{\left|f_{n}^{\prime}\left(a_{n}\right)\right|} \leq \frac{2}{n}$;

ii) $\left|f_{n}^{\prime}\left(z_{n}\right)\right| \geq\left|f_{n}^{\prime}\left(a_{n}\right)\right| \geq n^{3}$;

iii) If $x \in \overline{\mathbb{D}}(k)$ is such that $\left|x-z_{n}\right| \leq \frac{n}{\left|f_{n}^{\prime}\left(z_{n}\right)\right|}$, then

$$
\left|f_{n}^{\prime}(x)\right| \leq\left(1+\frac{1}{n}\right)\left|f_{n}^{\prime}\left(z_{n}\right)\right|
$$

It is clear that $z_{n} \rightarrow 0$. Now set $r_{n}=\frac{1}{\left|f_{n}^{\prime}\left(z_{n}\right)\right|}$, and pick $\rho_{n} \in k$ with $\left|\rho_{n}\right|=r_{n}$. We see that $r_{n} \leq \frac{1}{n^{3}}$, and hence $\rho_{n} \rightarrow 0$. Each map $g_{n}(z):=$ $f_{n}\left(z_{n}+\rho_{n} z\right)$ is hence defined on $\mathbb{D}(0 ; n)$. Fix some $R>0$ and pick $z \in \overline{\mathbb{D}}(R)$. We compute using Lemma 5.3 .

$$
\begin{aligned}
\left|g_{n}^{\prime}(z)\right| & \leq R^{2} \cdot r_{n} \cdot\left|f_{n}^{\prime}\left(z_{n}+\rho_{n} z\right)\right| \leq \\
& \leq R^{2} \cdot r_{n}\left(1+\frac{1}{n}\right)\left|f_{n}^{\prime}\left(z_{n}\right)\right|=R^{2}\left(1+\frac{1}{n}\right) .
\end{aligned}
$$

The Fubini-Study derivative of the maps $g_{n}$ is thus uniformly bounded on compact sets.

Clearly, $\left|g_{n}^{\prime}(0)\right|=\left|\rho_{n}\right| \cdot\left|f_{n}^{\prime}\left(z_{n}\right)\right|=1$ for all $n \in \mathbb{N}$.

\section{FurTher Notions OF HYPERBolicity}

In an attempt to obtain hyperbolicity results analogous to complex ones, we may consider other notions of hyperbolicity. As a first step, we compare the topologies on the set of rigid points induced by different semi distances.

6.1. The projective distance. Recall that for any two points given in homogeneous coordinates by $x=\left[x_{0}, \cdots, x_{N}\right], y=\left[y_{0}, \cdots, y_{N}\right] \in \mathbb{P}_{k}^{N}$, one defines their projective distance as

$$
d_{\mathbb{P}}(x, y)=\frac{\max \left|x_{i} y_{j}-x_{j} y_{i}\right|}{\max \left|x_{i}\right| \max \left|y_{j}\right|} .
$$


By continuity, one can extend this definition to the whole Berkovich projective space $\mathbb{P}^{N \text {,an }}$. This leads to the definition of discs in the projective space. We denote by $B_{d_{\mathbb{P}}}(x ; R)$ the open polydisk for the projective distance centered at $x$ and of radius $R$.

Observe that $d_{\mathbb{P}}(x, y) \leq 1$ for every $x, y \in \mathbb{P}^{N \text {,an }}$. Hence, if $R \geq 1$ one has that $B_{d \mathbb{P}}(x ; R)=\mathbb{P}^{N, \text { an }}$.

Lemma 6.1. Let $f: \mathbb{D} \rightarrow \mathbb{P}^{N \text {,an }}$ be an analytic map. Then,

$$
\sup _{x \in \mathbb{D}}\left|f^{\prime}(x)\right| \leq \sup _{x, y \in \mathbb{D}} \frac{d_{\mathbb{P}}(f(x), f(y))}{|x-y|} .
$$

Proof. Fix some point rigid point $x \in \mathbb{D}$. Using the Taylor series of $f$, we have that for $y$ close to $x$,

$$
\begin{aligned}
\left|\begin{array}{ll}
f_{i}(x) & f_{i}(y) \\
f_{j}(x) & f_{j}(y)
\end{array}\right| & =\left|\begin{array}{ll}
f_{i}(x) & f_{i}(x)+f_{i}^{\prime}(x)(x-y)+O\left((x-y)^{2}\right) \\
f_{j}(x) & f_{j}(x)+f_{j}^{\prime}(x)(x-y)+O\left((x-y)^{2}\right)
\end{array}\right|= \\
& =|x-y| \begin{array}{ll}
f_{i}(x) & f_{i}^{\prime}(x) \\
f_{j}(x) & f_{j}^{\prime}(x)
\end{array} \mid+O\left((x-y)^{2}\right) .
\end{aligned}
$$

This means that

$$
\left|f^{\prime}(x)\right|=\lim _{y \rightarrow x} \frac{d_{\mathbb{P}}(f(x), f(y))}{|x-y|},
$$

and thus $\left|f^{\prime}(x)\right| \leq \sup _{y \in \mathbb{D}} \frac{d_{\mathbb{P}}(f(x), f(y))}{|x-y|}$.

6.2. Topology induced by the projective distance. Let $X$ be a projective variety defined over a field $k$ of zero characteristic that is algebraically closed. Fixing an embedding of $X$ into some projective space, we obtain a distance function on $X(k)$ induced by the pull-back of the Fubini-Study distance. It is a fundamental fact that any two embeddings $X \rightarrow \mathbb{P}^{N \text {, an }}$ and $X \rightarrow \mathbb{P}^{M \text {,an }}$ induce equivalent distances on $X(k)$, see e.g. Gri15, Proposition 4.3].

Our aim is to prove that on the set of rigid points of $X$ the topology induced by $d_{\mathbb{P}}$ agrees with the Berkovich topology.

Proposition 6.2. Let $X$ smooth projective variety and fix an embedding $X \rightarrow \mathbb{P}^{N \text {,an }}$.

Then the Berkovich topology on the set of rigid points of $X$ agrees with the one induced by the projective distance $d_{\mathbb{P}}$.

To this end, we consider first the affinoid case.

Recall that given two points $z=\left(z_{1}, \ldots, z_{N}\right), w=\left(w_{1}, \ldots, w_{N}\right)$ in $\overline{\mathbb{D}}^{N}(k)$, the usual distance is given by

$$
d_{\mathbb{D}}(z, w)=\max _{1 \leq i \leq N}\left|z_{i}-w_{i}\right| .
$$

Lemma 6.3. Let $X$ be a strictly $k$-affinoid space and fix a closed immersion $X \rightarrow \overline{\mathbb{D}}^{N}$. The Berkovich topology on the set of rigid points of $X$ agrees with the one induced by the usual distance $d_{\mathbb{D}}$. 
Proof. Pick a rigid point $x \in X$ and fix some positive number $\epsilon$. The open ball $B_{d_{\mathbb{D}}}(x ; \epsilon)$ for the distance $d_{\mathbb{D}}$ centered at $x$ of radius $\epsilon$ can be expressed as the following finite intersection:

$$
B_{d_{\mathbb{D}}}(x ; \epsilon)=\bigcap_{i=1}^{N}\left\{z \in X(k):\left|\left(T_{i}-x_{i}\right)(z)\right|<\epsilon\right\} .
$$

For every $1 \leq i \leq N$, the set $\left\{z \in X(k):\left|\left(T_{i}-x_{i}\right)(z)\right|<\epsilon\right\}$ is an open set for the Berkovich topology.

Conversely, pick any Berkovich open set $U$ in $X(k)$. We may assume that $U$ is a finite intersection of sets of the form $\left\{x \in X(k): r_{i}<\left|f_{i}(x)\right|<s_{i}\right\}$ for some analytic function $f_{i} \in \mathcal{O}\left(\overline{\mathbb{D}}^{N}\right)$ and some positive real numbers $r_{i}$ and $s_{i}$. Recall that for any $z, w \in X(k)$ the following inequality holds:

$$
\left|f_{i}(z)-f_{i}(w)\right| \leq\left\|f_{i}\right\| d_{\mathbb{D}}(z, w),
$$

where $\|$.$\| denotes the norm on the Tate algebra \mathcal{T}_{N}$. As a consequence,

$$
U \cap X(k)=\bigcap_{i} \bigcup_{x \in U \cap X(k)} B_{d_{\mathbb{D}}}\left(x ; \frac{\min \left\{|| f_{i}(x)|-r|,|| f_{i}(x)|-s|\right\}}{\left\|f_{i}\right\|}\right),
$$

and the result follows.

Proof of Proposition 6.2. Pick a rigid point $x \in X$ and fix some positive real number $\epsilon<1$. The open ball $B_{d_{\mathbb{P}}}(x ; \epsilon)$ for the projective distance can be expressed as a finite intersection of open sets for the Berkovich topology as follows:

$$
B_{d_{\mathbb{P}}}(x ; \epsilon)=\bigcap_{\substack{0 \leq i, j \leq N \\ i \neq j}}\left\{y \in X(k):\left|\left(x_{i} T_{j}-x_{j} T_{i}\right)(y)\right|<\epsilon\right\} .
$$

The converse follows from the fact that the projective space can be covered by a finite number of Berkovich polydisks.

Given a projective variety $X$, we may consider the semi distance $d_{\mathrm{CK}}^{\prime}:=$ $\min \left\{1, d_{\mathrm{CK}}\right\}$. We now compare it with the projective distance.

Proposition 6.4. Let $X$ be a smooth projective variety. For any rigid point $x \in X$, there exists an open neighbourhood $U$ of $x$ and a positive constant $C$ such that $d_{\mathrm{CK}}^{\prime} \leq C d_{\mathbb{P}}$ on $U(k)$.

Proof. Denote by $M$ the dimension of $X$. Pick a rigid point $x \in X$ and fix an analytic map $\varphi: \mathbb{D}^{M} \rightarrow X$ that is an isomorphism on its image, sending some $z \in \mathbb{D}^{M}$ to $x$. Set $U:=\varphi\left(\mathbb{D}^{M}\right)$. Embed $X$ in some projective space $\mathbb{P}^{N \text {,an }}$. By Proposition 6.2, we may choose a positive number $\epsilon$ such that $B_{d_{\mathbb{P}}}(x ; \epsilon) \cap X(k)$ is contained in $U$.

After maybe reducing the polydisk $\mathbb{D}^{M}$, we may assume that $U$ is contained in some fixed unit polydisk $\overline{\mathbb{D}}^{N} \subset \mathbb{P}^{N \text {,an }}$. Thus, the projective distance agrees with the usual distance on $U$. Notice that the map $\varphi$ is given by $\varphi=\left(\varphi_{1}, \ldots, \varphi_{N}\right)$, where every $\varphi_{i} \in \mathcal{T}_{M}$ has coefficients bounded by 1 , for 
$1 \leq i \leq N$. Given any rigid point $y \in \overline{\mathbb{D}}^{N}$ with $y=\varphi(w)$ for some $w \in \mathbb{D}^{M}$, we have that

$$
d_{\mathbb{P}}(x, y)=d_{\mathbb{P}}(\varphi(z), \varphi(w))=\max _{1 \leq i \leq N}\left|\varphi_{i}(z)-\varphi_{i}(w)\right| .
$$

For distinct $z, w \in \mathbb{D}^{M}(k)$, consider the real-valued function

$$
\Theta(z, w)=\frac{d_{\mathbb{P}}(\varphi(z), \varphi(w))}{d_{\mathrm{CK}}^{\prime}(z, w)} .
$$

This function is strictly positive. By the previous equation, we know that $\Theta(z, w)=\frac{\max _{i \leq N}\left|\varphi_{i}(z)-\varphi_{i}(w)\right|}{\max _{j \leq M}\left|z_{j}-w_{j}\right|}$. The Taylor series development of each component $\varphi_{i}$ implies that for any $z, w \in \mathbb{D}^{M}(k)$, we may write $\varphi_{i}(w)-\varphi_{i}(z)=$ $\sum_{j=1}^{M}\left(z_{j}-w_{j}\right) \partial_{j} \varphi_{i}(z)+O\left(\sum_{1 \leq j \leq M}\left|z_{j}-w_{j}\right|^{2}\right)$, where $\partial_{j} \varphi_{i}(z)$ denotes the partial derivative of $\varphi_{i}$ with respect to the $j$-th component. Using this observation, we may extend the function $\Theta$ continuously to the diagonal by setting

$$
\Theta(z, z)=\lim _{w \rightarrow z} \Theta(z, w)=\max _{\substack{1 \leq i \leq N \\ 1 \leq j \leq M}}\left|\partial_{j} \varphi_{i}(z)\right| .
$$

As $\varphi$ is an isomorphism on its image, not all the partial derivatives $\partial_{j} \varphi_{i}$ are zero at the same time, and so $\Theta$ is strictly positive on the whole $\mathbb{D}^{M}(k) \times$ $\mathbb{D}^{M}(k)$. We may so find a positive constant $C$ such that $\Theta(z, w) \geq C$ for every $z, w \in \mathbb{D}^{M}(k)$. As the Cherry-Kobayashi semi distance contracts analytic maps, we see that $d_{\mathrm{CK}}(\varphi(z), \varphi(w)) \leq d_{\mathrm{CK}}(z, w)$. Thus, $d_{\mathrm{CK}}^{\prime}(\varphi(z), \varphi(w)) \leq$ $C d_{\mathbb{P}}(\varphi(z), \varphi(w))$.

6.3. Proof of Theorem A. We consider the following notion of hyperbolicity that arises naturally from the Cherry-Kobayashi semi distance:

Definition 6.5. Let $X$ be a smooth projective variety defined over an algebraically closed non-Archimedean complete field. The variety $X$ is strongly Cherry hyperbolic if the semi distance $d_{\mathrm{CK}}$ defines the same topology as the projective distance on rigid points.

We shall see in Theorem $\mathrm{A}$ that this notion is stronger than that of Cherry hyperbolicity. If $X$ is a hyperbolic complex analytic space, Barth showed that the Kobayashi metric defines the topology of $X$, see [Lan87, Theorem $\S I .2 .3]$.

Proof of Theorem $A$, i) $\Rightarrow$ ii): Let us first show that $X$ is Cherry hyperbolic. Pick any point $x \in X(k)$. We shall prove that $d_{\mathrm{CK}}(x, y)>0$ for all $y \in X(k)$ different from $x$.

By Corollary 6.2, the topology induced by $d_{\mathbb{P}}$ agrees with the Berkovich topology. Our assumption i) thus implies the existence of $\epsilon>0$ and a constant $C>0$ such that $d_{\mathrm{CK}}^{\prime}\left(x_{1}, x_{2}\right) \geq C d_{\mathbb{P}}\left(x_{1}, x_{2}\right)$ whenever

$$
\max \left\{d_{\mathbb{P}}\left(x, x_{1}\right), d_{\mathbb{P}}\left(x, x_{2}\right)\right\} \leq \epsilon .
$$


If $y$ is such that $d_{\mathbb{P}}(x, y) \leq \epsilon$, then $d_{\mathrm{CK}}^{\prime}(x, y) \geq C d_{\mathbb{P}}(x, y)>0$ as required. Suppose now that $d_{\mathbb{P}}(x, y)>\epsilon$, and pick any Kobayashi chain joining $x$ and $y$. We get a finite set of analytic maps $f_{l}: \overline{\mathbb{D}} \rightarrow X$ and points $z_{l} \in \overline{\mathbb{D}}(k)$, $l=1, \cdots, m$ such that $f_{1}(0)=x, f_{l}\left(z_{l}\right)=f_{l+1}(0)$ for $l=1, \cdots, m-1$ and $f_{m}\left(z_{m}\right)=y$. We shall prove that $\left|z_{l}\right| \geq C \epsilon / 4$ for some $l$, which proves that $d_{\mathrm{CK}}(x, y) \geq C \epsilon / 4$.

For each $l$, consider the function $d_{l}(t):=d_{\mathbb{P}}\left(x, f_{l}(t)\right)$. This is a continuous function on the whole disk $\overline{\mathbb{D}}$. Since all closed disks $\overline{\mathbb{D}}\left(0 ;\left|z_{l}\right|\right)$ are connected, the subset of the real line $\cup_{l=1}^{m} d_{l}\left(\overline{\mathbb{D}}\left(0 ;\left|z_{l}\right|\right)\right)$ is also connected. As $d_{1}(0)=0$ and $d_{m}\left(z_{m}\right)>\epsilon$, we may find an integer $l$ and a point $\tau \in \overline{\mathbb{D}}\left(0 ;\left|z_{l}\right|\right)$ such that $d_{l}(\tau)=\epsilon / 2$. By density of rigid points in the open disk, we may find $t \in \overline{\mathbb{D}}\left(0 ;\left|z_{l}\right|\right)(k)$ such that $d_{l}(t) \in(\epsilon / 4,3 \epsilon / 4)$. We get that $\left|z_{l}\right| \geq|t| \geq$ $d_{\mathrm{CK}}\left(x, f_{l}(t)\right) \geq C \epsilon / 4$.

We now prove that the two topologies induced by $d_{\mathrm{CK}}^{\prime}$ and $d_{\mathbb{P}}$ are the same. This amounts to checking that converging sequences for one topology are converging for the other one. Suppose first that $d_{\mathbb{P}}\left(x_{n}, x\right) \rightarrow 0$. Then for sufficiently large $n$ we have that $x_{n}$ lies in a neighbourhood of $x$ where $d_{\mathrm{CK}}^{\prime}$ is equivalent to $d_{\mathbb{P}}$, hence $d_{\mathrm{CK}}^{\prime}\left(x_{n}, x\right) \rightarrow 0$.

Suppose next that $d_{\mathrm{CK}}^{\prime}\left(x_{n}, x\right) \rightarrow 0$. Our arguments above show that for $n$ sufficiently large $x_{n}$ belongs to a neighbourhood of $x$ on which $d_{\mathrm{CK}}^{\prime}$ is equivalent to $d_{\mathbb{P}}$, so that again $d_{\mathbb{P}}\left(x_{n}, x\right) \rightarrow 0$.

ii) $\Rightarrow$ iii): Fix an embedding of $X$ in some analytic projective space $\mathbb{P}^{N \text {,an }}$. Suppose that the Fubini-Study derivative explodes at some point of $\mathbb{D}$. We apply Proposition 5.10 to find a sequence of analytic maps $g_{n}: \mathbb{D}(0 ; n) \rightarrow X$ satisfying $\left|g_{n}^{\prime}(0)\right|=1$ and with uniformly bounded Fubini-Study derivative on compact subsets of $\mathbb{A}^{1, \text { an }}$.

Denote by $d_{n}=\operatorname{diam}\left(g_{n}(\mathbb{D})\right)$. If $d_{n}$ tends to zero as $n$ goes to infinity, then after maybe extracting a subsequence all the $g_{n}(\mathbb{D})$ are contained in some fixed ball of $\mathbb{P}^{N \text {,an }}$. Schwarz' lemma implies that the derivative at zero is strictly smaller than 1 , contradicting the fact that $\left|g_{n}^{\prime}(0)\right|=1$. Thus, we may assume that there exists some $\epsilon>0$ such that $d_{n}>\epsilon$ for every $n \in \mathbb{N}$. In particular, for every $n$ there are rigid points $w_{n}, z_{m} \in \mathbb{D}$ such that $d_{\mathbb{P}}\left(g_{n}\left(w_{n}\right), g_{n}\left(z_{n}\right)\right) \geq \frac{\epsilon}{2}$. However, $d_{\mathrm{CK}}\left(g_{n}\left(w_{n}\right), g_{n}\left(z_{n}\right)\right) \leq d_{\mathrm{CK}}\left(w_{n}, z_{n}\right) \leq \frac{1}{n}$, since $g_{n}$ is defined on $\mathbb{D}(0 ; n)$, and so the distances $d_{\mathrm{CK}}$ and $d_{\mathbb{P}}$ cannot be equivalent.

iii) $\Rightarrow$ ii): Suppose that the Fubini-Study derivative of all the analytic maps $f: \mathbb{D} \rightarrow X$ is uniformly bounded on some open disk $\mathbb{D}(0 ; r)$ by some positive constant $C$. It suffices to show that given any rigid points $x_{n}, x$ in $X$ such that $d_{\mathrm{CK}}\left(x_{n}, x\right)$ tends to $0, d_{\mathbb{P}}\left(x_{n}, x\right) \rightarrow 0$ as $n$ goes to infinity.

For every $n$, consider a Kobayashi chain $f_{l}^{n}: \mathbb{D} \rightarrow X, l=1, \cdots, N_{n}$, joining $x_{n}$ and $x$ of length $r_{n}<r$ and such that $r_{n} \rightarrow 0$. By Lemma 5.8, we see that $f_{l}^{n}\left(\mathbb{D}\left(0 ; r_{n}\right)\right) \subseteq \mathbb{D}^{N}\left(f_{l}^{n}(0) ; r_{n} C\right)$. For every fixed $n \in \mathbb{N}^{*}$, these polydisks have nonempty intersection by definition of Kobayashi chain and 
have the same radius, and so they must be the same. Hence, $d_{\mathbb{P}}\left(x_{n}, x\right)$ tends to 0 .

iii) $\Rightarrow$ i): Let $r>0$ and $C>0$ be constants such that

$$
\sup _{\operatorname{Mor}_{k}(\mathbb{D}, X)} \sup _{\mathbb{D}(0 ; r)}\left|f^{\prime}(z)\right| \leq C .
$$

After maybe reducing the radius $r$, we may assume that $d_{\mathrm{CK}}^{\prime} \leq d_{\mathbb{P}}$ on $\mathbb{D}(0 ; r)$ by Proposition 6.4. Pick any two rigid points $x, y \in X$. Assume that $d_{\mathrm{CK}}(x, y) \leq \frac{r}{2}$. Let $C_{n}$ be a sequence of Kobayashi chains joining $x$ and $y$ of length $d_{n}$ and such that $\lim _{n} d_{n}=d_{\mathrm{CK}}(x, y)$. Recall that each chain $C_{n}$ is given by analytic maps $f_{1}^{(n)}, \ldots, f_{N_{n}}^{(n)}: \mathbb{D} \rightarrow X$ and rigid points $z_{1}^{(n)}, \ldots, z_{N_{n}}^{(n)}$ in $\mathbb{D}$ satisfying the appropritate equalities. For sufficiently large $n$ we may assume that

$$
d_{\mathrm{CK}}(x, y) \leq d_{n}<2 \cdot d_{\mathrm{CK}}(x, y)=r,
$$

and in particular we see that $\left|z_{l}^{(n)}\right|<r$ for every $n \gg 0$ and every $1 \leq$ $l \leq N_{n}$. As a consequence of Lemma 5.8 , we see that $f_{l}^{(n)}\left(\overline{\mathbb{D}}\left(0 ;\left|z_{l}^{(n)}\right|\right)\right) \subseteq$ $\mathbb{D}^{N}\left(f_{l}^{(n)}(0) ;\left|z_{l}^{(n)}\right| C\right)$. Thus, for every $n \in \mathbb{N}$ we have

$$
d_{\mathbb{P}}(x, y) \leq C \cdot d_{n}<2 C \cdot d_{\mathrm{CK}}(x, y),
$$

concluding the proof.

Remark 6.6. Residue characteristic 0 is used for the implications iii) $\Rightarrow$ i) and iii) $\Rightarrow$ ii).

Proposition 6.7. Let $X$ be a smooth projective variety defined over an algebraically closed non-Archimedean complete field $k$ of residue characteristic zero. If the Fubini-Study derivative of $\operatorname{Mor}_{k}(\mathbb{D}, X)$ is uniformly bounded in a neighbourhood of every rigid point, then the family $\operatorname{Mor}_{k}(\mathbb{D}, X)$ is normal at every rigid point.

Proof. Embed $X$ in some projective space $\mathbb{P}^{N \text {,an }}$. It suffices to prove the assertion for $z=0$ in $\mathbb{D}$. Assume first that $U$ is a neighbourhood of 0 on which there exists a positive constant $C$ such that

$$
\sup _{\operatorname{Mor}_{k}(\mathbb{D}, X)} \sup _{U}\left|f^{\prime}(z)\right| \leq C<+\infty .
$$

Pick any sequence of analytic maps $f_{n}: \mathbb{D} \rightarrow X$. Since $\mathbb{P}^{N \text {,an }}$ can be covered by a finite number of closed polydisks isomorphic to $\overline{\mathbb{D}}^{N}$, we see that, after maybe to extracting a subsequence and rescaling the image, the points $f_{n}(0)$ converge to a point in $\overline{\mathbb{D}}^{N}\left(0 ; \frac{1}{2}\right)$, as $\mathbb{P}^{N \text {,an }}$ is sequentially compact Poi13].

Now let $\eta_{0, r} \in U$ be the point corresponding to the closed all $\bar{B}(0 ; r)$ in $k$. It follows from Lemma 5.8 that $\operatorname{diam} f_{n}\left(\eta_{0, r}\right) \leq r \cdot C$ for all $n$. Choose $r>0$ such that $r \leq \frac{1}{2 C}$ and set $U^{\prime}=\mathbb{D}(0 ; r)$. By continuity, $f_{n}\left(U^{\prime}\right) \subseteq$ $\overline{\mathbb{D}}^{N}\left(0 ; \frac{1}{2}\right)$, and by Theorem 2.12 there exists a subsequence converging on $U^{\prime}$ to a continuous map. 
6.4. Proof of Theorem $\mathbf{B}$, The equivalence between i) and v) was proved in Che96. We provide a new proof of the fact that every smooth projective curve with positive genus is Cherry hyperbolic.

iv) $\Rightarrow$ i): Pick any $|\lambda|>1$ and consider the sequence of analytic maps $f_{n}(z)=(\lambda z)^{n}$ from $\mathbb{D}$ to $\mathbb{P}^{1, \text { an }}$. As explained at the beginning of this chapter, no subsequence of $f_{n}$ has a continuous limit, and thus the family $\operatorname{Mor}_{k}\left(\mathbb{D}, \mathbb{P}^{1, \text { an }}\right)$ is not normal.

i) $\Rightarrow$ iv): Assume that $X$ has positive genus. Recall from $\$ 3$ that if $X$ is a smooth projective curve with positive genus, then $S^{\text {an }}(X)$ is nonempty. The set $X \backslash \mathrm{S}^{\text {an }}(X)$ is a disjoint union of infinitely many open disks.

Let $f_{n}: \mathbb{D} \rightarrow X$ be a sequence of analytic maps. By Ber90, Theorem 4.5.3], the image of each map $f_{n}$ does not intersect $S^{\text {an }}(X)$.

If the image of infinitely many maps $f_{n}$ is contained in the same connected component of $X \backslash \mathrm{S}^{\text {an }}(X)$, then we may find a subsequence $f_{n_{j}}$ avoiding some fixed connected component of the complement of $\mathrm{S}^{\mathrm{an}}(X)$. Hence, by Lemma 3.6 the maps $f_{n_{j}}$ take values in a fixed affinoid domain of $X$ and so they converge pointwise to some continuous map by Theorem 2.12 .

Assume next that at most finitely many $f_{n}(\mathbb{D})$ are contained in the same connected component of $X \backslash \mathrm{S}^{\mathrm{an}}(X)$. If $\mathrm{S}^{\text {an }}(X)$ consists only of one point $\eta_{X}$, this means that the sequence $f_{n}$ converges pointwise to the constant map $\eta_{X}$. Suppose otherwise $\mathrm{S}^{\mathrm{an}}(X)$ is not a singleton. Denoting by $r_{X}: X \rightarrow$ $\mathrm{S}^{\text {an }}(X)$ the usual retraction map, we consider the composition $y_{n}:=r_{X} \circ f_{n}$. Thus, each map $y_{n}$ is constant. By compactness of $S^{\text {an }}(X)$ we may find a subsequence $\left\{y_{n_{j}}\right\}$ converging to some point $y \in \mathrm{S}^{\mathrm{an}}(X)$.

Fix an open neighbourhood $V \ni y$. By [Duc14, Théorème 4.5.4], we are reduced to the following possibilities for $V$. If $y$ is a type III point, then $V$ is isomorphic to an open annulus whose skeleton is contained in $\mathrm{S}^{\text {an }}(X)$. Otherwise, if $y$ has type II then $V \backslash\{y\}$ is the disjoint union of infinitely many open disks and finitely many open annuli. In particular, the intersection of the skeleton of $V$ and $\mathrm{S}^{\text {an }}(X)$ is nonempty. Pick any $z \in \mathbb{D}$. For sufficiently large $n_{j}$, the points $f_{n_{j}}(z)$ lie in $V$. Thus, the subsequence $\left\{f_{n_{j}}\right\}$ converges pointwise to the constant map $f \equiv y$.

i) $\Rightarrow$ iii): Let $X$ be a curve of positive genus. We show that the FubiniStudy derivative of every map from $\mathbb{D}$ to $X$ is bounded. Let $f: \mathbb{D} \rightarrow X$ be an analytic map. By [Ber90, Theorem 4.5.3], the image of $f$ is contained in some connected component of the complement of $\mathrm{S}^{\mathrm{an}}(X)$, i.e. in some open subset $V$ of $X$ analytically isomorphic to $\mathbb{D}$. This implies that the Fubini-Study derivative of $f$ is bounded by 1 on the whole disk.

iii) $\Leftrightarrow$ ii): This equivalence was shown in Theorem A.

ii) $\Rightarrow \mathrm{v}$ ): This implication follows from Theorem A.

\section{Curves With non-negative Euler Characteristic}

In this section we prove one of the implications of Theorem C. 
Proposition 7.1. Let $X$ be a curve with non-negative Euler characteristic. Then there exists a one-dimensional basic tube $U$ such that the family $\operatorname{Mor}_{k}(U, X)$ is not normal.

Our proof follows [FKT12, Theorem 5.4] and uses an equidistribution result for non-Archimedean elliptic curves from [Pet09].

Proof. Recall that a smooth algebraic curve satisfies $\chi(X) \geq 0$ if and only if it is isomorphic to one of the following models:

(1) $\mathbb{P}^{1, \text { an }}, \mathbb{A}^{1 \text {,an }}, \mathbb{A}^{1 \text {,an }} \backslash\{0\}$;

(2) an elliptic curve.

We claim that for any $\rho>1$ the family of analytic maps

$$
\operatorname{Mor}_{k}\left(A\left(\frac{1}{\rho}, \rho\right), \mathbb{A}^{1, \text { an }} \backslash\{0\}\right)
$$

is not normal. To see this, consider the sequence $f_{n}(z)=z^{n}$ from $A\left(\frac{1}{\rho}, \rho\right)$ to $\mathbb{A}^{1, \text { an }} \backslash\{0\}$. Observe that $f_{n}\left(x_{g}\right)=x_{g}$ for all $n \in \mathbb{N}$, whereas $f_{n}(z) \rightarrow 0$ for any $|z|<1$. It follows that no limit map of $f_{n}$ can be continuous at the Gauss point. As a consequence of the definition of normality given at the begining of this chapter for families of maps whose target is a smooth algebraic curve, this proves the proposition for all cases in the first item.

Suppose now that $X$ is an elliptic curve. Consider the map $f: X \rightarrow$ $X$ induced by the multiplication by 2 . Pick any point $x_{0} \in \mathrm{S}^{\text {an }}(X)$, and suppose by contradiction that the family of the iterates $\left\{f^{n}\right\}$ is normal on a neighborhood $U$ of $x_{0}$. Assume that the subsequence $f^{n_{j}}$ converges on $U$ to a continuous function $g: U \rightarrow X$.

Choose any fixed rigid point $y \in X$ for $f$. By [Pet09, Theorem 1] the sequence of probability measures $4^{-n}\left(f^{n}\right)^{*} \delta_{y}$ converges to a probability measure $\mu$ whose support is equal to $\mathrm{S}^{\text {an }}(X)$, hence contains $x_{0}$. We may thus find a sequence of rigid points $y_{n} \rightarrow x_{0}$ such that $f^{n}\left(y_{n}\right)=y$. Observe that $y_{m} \in U$ for sufficiently large $m$, thus $g\left(y_{m}\right)=\lim _{n_{j}} f^{n_{j}}\left(y_{m}\right)=y$, for all $m \in \mathbb{N}$.

But $f$ leaves the skeleton of $X$ invariant, hence $y=g\left(x_{0}\right) \in \mathrm{S}^{\text {an }}(X)$ which gives a contradiction.

\section{Analytic maps on SPECial domains}

In this section, we study the normality of the family of analytic maps taking values in a smooth projective curve $X$ having only one node that do not have good reduction, i.e. whose skeleton contains points different from the node. Our discussion is based on the study of three fundamental families of one-dimensional basic tubes: open disks, open annuli and starshaped domains. 


\subsection{Analytic maps avoiding a type II point.}

Proposition 8.1. Let $X$ be a smooth irreducible projective curve and $U$ any smooth connected curve. Let $\mathcal{F}$ be a family of analytic maps from $U$ to $X$.

If there exists a type II point $\eta \in X$ such that $\eta \notin f(U)$ for every $f \in \mathcal{F}$, then there exists an affinoid covering $\left(X_{1}, X_{2}\right)$ of $X$ such that for every $f \in$ $\mathcal{F}$, the image $f(U)$ is contained either in $X_{1}$ or in $X_{2}$.

Moreover, the affinoid cover $\left(X_{i}\right)$ is independent of $U$.

Corollary 8.2. Let $X$ be a smooth irreducible projective curve of genus at least 2 and $U$ be an open disk or an open annulus. Then, there exists a finite affinoid cover $\left(X_{i}\right)$ of $X$ such that the image of every analytic map $f: U \rightarrow X$ is contained in some affinoid $X_{i}$.

Moreover, the affinoid cover $\left(X_{i}\right)$ is independent of $U$.

Corollary 8.3. Let $X$ be a smooth irreducible projective curve having at least two nodes and let $U$ be any smooth connected boundaryless curve. Then, there exists a finite affinoid cover $\left(X_{i}\right)$ of $X$ and a locally finite open cover $\left(U_{j}\right)$ of $U$ by basic tubes such that for every analytic map $f: U \rightarrow X$ and every element of the cover $U_{j}$, the image $f\left(U_{j}\right)$ is contained in some affinoid $X_{i}$.

Moreover, the affinoid cover $\left(X_{i}\right)$ is independent of $U$.

Proof of Proposition 8.1. Let $X$ be any smooth irreducible projective curve and $U$ any smooth connected curve. Let $\mathcal{F}$ be a family of analytic maps in $\operatorname{Mor}_{k}(U, X)$ whose images avoid some type II point $\eta \in X$.

The image of every $f \in \mathcal{F}$ is contained in some connected component of $X \backslash\{\eta\}$. We may thus pick any two distinct connected components $B_{1}, B_{2}$ of $X \backslash\{\eta\}$. Then, $X_{1}:=X \backslash B_{1}$ and $X_{2}=X \backslash B_{2}$ are affinoid domains of $X$ by Lemma 3.6, and $\left(X_{1}, X_{2}\right)$ is a cover of $X$ safisfying the required property.

Proof of Corollary 8.2. Let $X$ be a smooth irreducible projective curve of genus at least two. The curve $X$ contains at least one node $\eta$ by (3.1). On the other hand, if $U$ is an open disk or an open annulus, then it has no nodes. By Lemma 3.11, every analytic map $f: U \rightarrow X$ avoids $\eta$. The result follows from Proposition 8.1 .

Proof of Corollary 8.3. Let $U$ be any smooth irreducible boundaryless curve. Recall that its set of nodes is discrete. Consider a locally finite open cover $\left(U_{j}\right)_{j \in J}$ of $U$, where each $U_{j}$ is either an open disk, an open annulus or a star-shaped domain. In particular, every basic tube $U_{j}$ contains at most one node.

Let $X$ be a smooth projective curve with at least two nodes, and denote $\mathrm{N}(X)=\left\{\eta_{1}, \ldots, \eta_{a}\right\}$. For every $1 \leq l \leq a$, let $B_{l}^{1}$ and $B_{l}^{2}$ be two distinct connected components of $X \backslash\left\{\eta_{l}\right\}$ that are isomorphic to an open disk. The sets $X_{l}^{1}=X \backslash B_{l}^{1}$ and $X_{l}^{2}=X \backslash B_{l}^{2}$ are affinoid domains of $X$ by Lemma

3.6. The sets $\left(X_{l}^{i}\right)_{i=1,2}$ form a finite affinoid cover of $X$. 
Fix some $U_{j}$. For every fixed $1 \leq l \leq a$, consider the family of analytic maps $\mathcal{F}_{l, j}=\left\{f: U \rightarrow X\right.$ analytic $\left.: \eta_{l} \notin f\left(U_{j}\right)\right\}$. By Lemma 3.11, we have that

$$
\bigcup_{\substack{1 \leq l \leq a \\ j \in J}} \mathcal{F}_{l, j}=\operatorname{Mor}_{k}(U, X) .
$$

We conclude by applying Proposition 8.1 to every family $\mathcal{F}_{l, j}$.

\subsection{Analytic maps into curves having only one node.}

Proposition 8.4. Let $X$ be a smooth irreducible projective curve over $k$ of genus at least 2 having a unique node $\eta_{X}$. Assume further that the first Betti number of the skeleton of $X$ is at least 1 . Let $U$ be a smooth connected boundaryless curve.

Then there exists a finite affinoid cover $\left(X_{i}\right)_{i \in I}$ of $X$ and a locally finite open cover $\left(U_{j}\right)_{j \in J}$ of $U$ by basic tubes such that for every analytic map $f: U \rightarrow X$ and every $j \in J$, the set $f\left(U_{j}\right)$ is contained in some affinoid domain $X_{i}$.

Moreover, the affinoid cover $\left(X_{i}\right)$ is independent of $U$.

Proof. Let $b:=b_{1}\left(\mathrm{~S}^{\mathrm{an}}(X)\right)>1$ be the first Betti number of the skeleton of $X$. As $b \geq 1$ and $X$ has only one node, the skeleton of $X$ consists of $b$ loops $C_{1}, \ldots, C_{b}$ passing through $\eta_{X}$, and so there are exactly $2 b$ non-discal tangent directions at $\eta_{X}$.

The curve $X$ may be decomposed as a disjoint union of $\left\{\eta_{X}\right\}$, open annuli $A_{1}, \ldots, A_{b}$ and infinitely many open disks by Lemma 3.5. Fix some $1 \leq$ $i \leq b$. Pick an isomorphism $\varphi_{i}: A\left(R_{i}, 1\right) \rightarrow A_{i}$ with $R_{i}<1$ and such that $\lim _{r \rightarrow 1} \varphi_{i}\left(\eta_{0, r}\right)=\lim _{r \rightarrow R_{i}} \varphi_{i}\left(\eta_{0, r}\right)=\eta_{X}$. Consider the type II point $x_{i}:=\varphi_{i}\left(\eta_{0, \sqrt{R_{i}}}\right)$, which lies on the loop $C_{i}$. Pick any connected component $B_{i}$ of $X \backslash\left\{x_{i}\right\}$ isomorphic to an open disk. The set $X_{i}:=X \backslash B_{i}$ is an affinoid domain of $X$ by Lemma 3.6 and contains the point $\eta_{X}$. The family $\left(X_{i}\right)_{1 \leq i \leq b}$ forms an affinoid cover of $X$.

Since $U$ is paracompact (cf. Theorem 3.1), it suffices to show that for every point $z \in U$ there exists an open neighbourhood $V_{0}$ of $z$ such that for every analytic map $f: U \rightarrow X$, there exists some affinoid domain $X_{i}$ in the cover of $X$ such that $f\left(V_{0}\right) \subset X_{i}$. Moreover, since the cases of disks and annuli have been treated separately in Corollary 8.2, obtaining an affinoid cover of $X$ similar to $\left(X_{i}\right)$, we may assume that $z \in \mathrm{N}(U)$. We aim to construct a star-shaped domain $V_{0} \subset U$ containing $z$ and such that every analytic map $f: V_{0} \rightarrow X$ sends $V_{0}$ to some affinoid domain $X_{i}$.

Let $V$ be the connected component of the complement of $\mathrm{N}(U) \backslash\{z\}$ in $U$ containing $z$. It is a star-shaped domain in $U$ containing $z$ whose only node $\eta_{V}$ is precisely $z$. Let $\left\{\vec{v}_{1}, \ldots, \vec{v}_{a}\right\}$ be the set of non-discal directions at $\eta_{V}$. Fix some $1 \leq j \leq a$ and let $I_{j}$ be the connected component of $S^{\text {an }}(V) \backslash\left\{\eta_{V}\right\}$ corresponding to the direction $\vec{v}_{j}$. It is isomorphic to an open segment. The set $U\left(\vec{v}_{j}\right)$ is isomorphic to an open annulus whose skeleton is precisely $I_{j}$. 
We fix an isomorphism $\psi_{j}: A\left(\rho_{j}, 1\right) \rightarrow U\left(\vec{v}_{j}\right)$ with $\rho_{j}<1$ and such that $\psi_{j}$ extends continuously to the Gauss point $x_{g}$ satisfying $\psi_{j}\left(x_{g}\right)=\eta_{V}$.

Pick any analytic map $f: U \rightarrow X$. If $f(V)$ avoids the node $\eta_{X}$, then it is contained in some annulus $A_{i}$ or in some connected component of the complement of $\eta_{X}$ that is isomorphic to an open disk, hence in some affinoid domain $X_{i}$.

Assume otherwise that $f$ maps the unique node $z=\eta_{V} \in V$ to $\eta_{X}$.

Consider the star-shaped domain $V_{0}$ contained in $V$ obtained by reducing every segment $I_{j}$ in such a way that the resulting segment $I_{j}^{0}$ is isomorphic to $\left(\rho_{j}^{0}, 1\right)$ with $\rho_{j}^{0} \geq \max _{1 \leq j \leq a} \sqrt{\rho_{j}}$. We claim that $x_{i}$ does not belong to $f\left(I_{j}^{0}\right)$ and that $f\left(V_{0}\right) \subseteq X_{i}$ for some index $i$.

Recall that the tangent map $d f\left(\eta_{V}\right): T_{\eta_{V}} V \rightarrow T_{\eta_{X}} X$ is a rational map on the residue curve at $\eta_{V}$ with values in the residue curve at $\eta_{X}$, which is surjective. The preimage of every non-discal direction at $\eta_{X}$ consists only of non-discal directions at $\eta_{V}$ by Lemma 3.8. We may thus choose $j$ such that $d f\left(\eta_{V}\right)\left(\vec{v}_{j}\right) \in T_{\eta_{X}} X$ is non-discal.

The restriction of $f$ to $U\left(\vec{v}_{j}\right)$ takes values in some annulus $A_{i} \subset X$, and so we may consider the composition $F_{i, j}=\varphi_{i}^{-1} \circ f \circ \psi_{j}: A\left(\rho_{j}, 1\right) \rightarrow$ $A\left(R_{i}, 1\right)$. Since $f\left(\eta_{V}\right)=\eta_{X}$, we see that $\lim _{r \rightarrow 1} F_{i, j}\left(\eta_{0, r}\right)=x_{g}$. Additionally, $F_{i, j}\left(\mathrm{~S}^{\mathrm{an}}\left(A\left(\rho_{j}, 1\right)\right) \subseteq \mathrm{S}^{\mathrm{an}}\left(A\left(R_{i}, 1\right)\right)\right.$ by Proposition 3.10 .

The map $F_{i, j}$ can be expanded into a Laurent series $F_{i, j}(z)=\sum_{n \in \mathbb{Z}} a_{n} z^{n}$. Consider the real function $\theta_{i, j}(r):=\max _{n \in \mathbb{Z}}\left\{\log \left|a_{n}\right|+n r\right\}$, defined on the open real interval $\left(\log \rho_{j}, 0\right)$. Since $F_{i, j}$ is an analytic function on an open annulus without zeroes, there exists an integer $n_{0} \in \mathbb{Z}$ such that the function $\theta_{i, j}$ is of the form $\theta_{i, j}(r)=\log \left|a_{n_{0}}\right|+n_{0} r$. As $F_{i, j}$ extends continuously to the Gauss point in $A\left(\rho_{j}, 1\right)$ with $\lim _{r \rightarrow 1} F_{i, j}\left(\eta_{0, r}\right)=x_{g}$, we see that $\left|a_{n_{0}}\right|=1$. It follows that $\theta_{i, j}$ extends continuously to the origin $0 \in \mathbb{R}$ with $\theta_{i, j}(0)=0$.

Observe that $\theta_{i, j}(r) \geq \log R_{i}$ for every $r \in\left(\log \rho_{j}, 0\right)$ by the definition of $F_{i, j}$. It follows that the graph of $\theta_{i, j}$ lies above the linear function $r \in$ $\left(\log \rho_{j}, 0\right) \mapsto \frac{\log R_{i}}{\log \rho_{j}} r$. In particular, we see that $n_{0} \leq \frac{\log R_{i}}{\log \rho_{j}}$. We conclude that $\theta_{i, j}(r)>\frac{1}{2} \log R_{i}$ as soon as $r>\log \sqrt{\rho_{j}}$. Notice that this condition does not depend on $R_{i}$.

Pick any $1>\rho_{j}^{0}>\max _{1 \leq j \leq a} \sqrt{\overline{\rho_{j}}}$ and reduce the segment $I_{j}$ into a segment $I_{j}^{0}$ such that the corresponding open subset $U\left(\vec{v}_{j}^{0}\right) \subset V$ is isomorphic to $\psi_{j}\left(A\left(\rho_{j}^{0}, 1\right)\right)$. The previous calculations show that the image under $f$ of the segment $I_{j}^{0}$ covers at most half the loop $C_{i}$ at $\eta_{X}$ starting with the direction $d f\left(\eta_{U}\right)\left(\vec{v}_{j}\right)$, avoiding the point $x_{i} \in X$. It follows that $f\left(U\left(\vec{v}_{j}^{0}\right)\right) \subseteq X_{i}$.

We may carry over this procedure to every non-discal direction at $\eta_{V}$, imposing that $\rho_{j}^{0}>\max _{1 \leq j \leq a} \sqrt{\rho_{j}}$ for every $j=1, \ldots, a$. Let $V_{0}$ be the resulting star-shaped domain in $U$, which contains the point $z$. We conclude that the restriction of any $f: U \rightarrow X$ to $V_{0}$ takes values in some affinoid domain $X_{i}$. 


\section{Proof of Theorem D}

From now on, we will suppose that the base field $k$ has zero residue characteristic.

9.1. De Franchis theorem. At several stages of the proofs of Theorems D and $\mathrm{C}$, we shall need the following (slight) improvement of the original De Franchis theorem that applies to nonproper curves. We refer for example to Tsu79 for a purely algebraic proof in a much more general context in arbitrary dimension.

Theorem 9.1. Let $\tilde{k}$ be any algebraically closed field of characteristic zero. Let $X$ and $Y$ be two smooth algebraic curves defined over $\tilde{k}$. Suppose that $\chi(X)<0$, with $X$ not necessarily proper. Then the set of regular maps from $Y$ to $X$ is finite.

9.2. The compact case. Let $X$ be a smooth projective curve of genus at least 2 not having good reduction. Recall that the latter condition means that its skeleton consists of more than one point. Since $g(X) \geq 2$, then we know that $\mathrm{N}(X)$ is nonempty.

Let $U$ be any smooth boundaryless curve. If $\mathrm{N}(X)$ consists of more than one point, then we conclude by Corollary 8.3 . Otherwise, we are exactly in the situation of Proposition 8.4 .

9.3. General algebraic case. Let $X$ be a smooth irreducible algebraic curve with negative Euler characteristic whose skeleton is not a singleton, and let $\bar{X}$ be the unique smooth projective curve such that there exists an open embedding $X \rightarrow \bar{X}$ with $\bar{X} \backslash X$ a finite set of rigid points. Let $U$ be any smooth connected boundaryless curve.

Our aim is to construct a finite affinoid cover $\left(\bar{X}_{i}\right)$ of $\bar{X}$ and a locally finite cover $\left(U_{j}\right)$ of $U$ such that for every analytic map $f: U \rightarrow X$ there exists some $\bar{X}_{i}$ with $f\left(U_{j}\right) \subseteq \bar{X}_{i}$.

Recall that the non-proper algebraic curves $X$ with negative Euler characteristic are $\mathbb{P}^{1, \text { an }}$ with at least three rigid points removed, and elliptic curves and curves with genus at least 2 with finitely many rigid points removed.

The case of $\mathbb{P}^{1 \text {,an }} \backslash\{0,1, \infty\}$ is treated in [FKT12, Proposition 3.2]. If $X$ is such that $\bar{X}$ does not have good reduction and its genus is greater than 1 , then we are reduced to the projective case, which has already been treated.

Therefore, it only remains to address the cases where $X$ is either an elliptic curve or a projective curve with good reduction $\bar{X}$ with a rigid point removed. The curve $X$ has exactly one node $\eta_{X}$, which is a branching point of the skeleton in the case where $\bar{X}$ has bad reduction and a point of positive genus if $\bar{X}$ has good reduction. We shall make no distinction in the genus of $\bar{X}$ when dealing with the good reduction case.

If $\bar{X}$ is an elliptic curve with bad reduction, then the skeleton of $X$ consists of a loop $C$ passing through $\eta_{X}$ and the segment joining $\eta_{X}$ and the unique point in $\bar{X} \backslash X$ with its endpoint removed. We are in a situation similar to 
that of Proposition 8.4. We obtain the following affinoid cover $(\bar{X})_{i=1,2}$ of $X$. Let $x \in \mathrm{S}^{\text {an }}(X)$ be any type II point on the loop $C \subset \mathrm{S}^{\text {an }}(X)$ different from $\eta_{X}$ and pick any connected component $B_{1}$ of $X \backslash\{x\}$ that is isomorphic to an open disk. We set $\bar{X}_{1}:=\bar{X} \backslash B_{1}$. Set $\bar{X}_{2}:=\bar{X} \backslash B_{2}$, where $B_{2}$ is a connected component of $X \backslash\left\{\eta_{X}\right\}$ isomorphic to a disk. We obtain a finite open cover of $U$ by basic tubes $U_{j}$ having at most one node.

Assume now that $\bar{X}$ is a curve with good reduction and genus at least 1 . There is only one non-discal direction $\vec{w}$ at $\eta_{X}$, and the set $U(\vec{w})$ is isomorphic to a punctured disk. Pick any analytic map $f: U \rightarrow X$.

Consider the following affinoid cover $\left(\bar{X}_{1}, \bar{X}_{2}\right)$ of $\bar{X}$. Pick an open subset $B_{1}$ of $\bar{X}$ isomorphic to an open disk and containing the unique point in $\bar{X} \backslash X$. Assume further that $B_{1} \cap X$ is strictly contained in $U(\vec{w})$ and set $\bar{X}_{1}:=\bar{X} \backslash B_{1}$. Let $B_{2}$ be a connected component of $X \backslash\left\{\eta_{X}\right\}$ isomorphic to a disk and set $\bar{X}_{2}:=\bar{X} \backslash B_{2}$.

If $f$ avoids the point $\eta_{X}$, then $f(U)$ is clearly contained either in $\bar{X}_{1}$ or in $\bar{X}_{2}$. We may thus assume that there exists some $\eta_{j} \in \mathrm{N}(U)$ such that $f\left(\eta_{j}\right)=\eta_{X}$.

Let $U_{j} \subset U$ be the connected component of the complement of $\mathrm{N}(U) \backslash\left\{\eta_{j}\right\}$ in $U$ containing $\eta_{j}$. The tangent map $d f\left(\eta_{j}\right)$ is surjective, and by Lemma 3.8 every preimage of $\vec{w}$ is non-discal. We may thus pick $\vec{v} \in T_{\eta_{j}} U$ non-discal such that $d f\left(\eta_{j}\right)(\vec{v})=\vec{w}$. Fix isomorphisms $\varphi: \mathbb{D} \backslash\{0\} \rightarrow U(\vec{w})$ and $\psi$ : $A(\rho, 1) \rightarrow U(\vec{v})$ with $\rho<1$. Assume further that both extend continuously to the Gauss point, with $\lim _{r \rightarrow 1} \varphi\left(\eta_{0, r}\right)=\eta_{X}$ and $\lim _{r \rightarrow 1} \psi\left(\eta_{0, r}\right)=\eta_{X}$.

The composition $F=\varphi^{-1} \circ f \circ \psi$ is so an analytic map on an open annulus with values in the punctured disk. Write $F(z)=\sum_{n \in \mathbb{Z}} a_{n} z^{n}$ and consider the real function $\theta(r)=\max _{n \in \mathbb{Z}}\left\{\log \left|a_{n}\right|+n r\right\}$ on the open interval $(\log \rho, 0)$. Since $F$ has no zeros, there exists an integer $n_{0}$ such that $\theta(r)=\log \left|a_{n_{0}}\right|+n_{0} r$ for all $r \in(\log \rho, 0)$. Moreover, $\left|a_{n_{0}}\right|=1$, as $F\left(x_{g}\right)=x_{g}$.

Consider the tangent map $d f\left(\eta_{j}\right): T_{\eta_{j}} U_{j} \rightarrow T_{\eta_{X}} X$. Both $T_{\eta_{j}} U_{j}$ and $T_{\eta_{X}} X$ are isomorphic to smooth projective curves $C_{U_{j}}$ and $C_{X}$ respectively over $\tilde{k}$ with a finite number of marked points, corresponding to the non-discal directions at $\eta_{j}$ and $\eta_{X}$ respectively. In particular, $C_{X}$ is a curve with one marked point and genus at least 1 . The inverse image under $d f\left(\eta_{j}\right)$ of the marked point in $C_{X}$ is contained in the set of marked points in $C_{U_{j}}$ by Lemma 3.8. Applying Theorem 9.1 to the curve $C_{X}$ with the marked point, we obtain that there are only finitely many possibilities for the tangent map $d f\left(\eta_{j}\right)$. As a consequence, the degree at every marked point in $C_{U_{j}}$ of the rational map $d f\left(\eta_{j}\right)$ is bounded. At the marked point corresponding to $\vec{v} \in T_{\eta_{j}} U_{j}$, this degree is precisely $n_{0}$, the slope of $\theta$, which is thus bounded.

Hence, after maybe reducing the basic tube $U_{j}$ we see that $f\left(U_{j}\right) \subset \bar{X}_{1}$. Repeating this procedure at every node of $U$, we obtain a locally finite open cover $\left(U_{j}\right)$ of $U$ consisting of open disks, open annuli and star-shaped domains satisfying the required property. 
Remark 9.2. The previous arguments in the case where $\bar{X}$ has good reduction apply verbatim to $\mathbb{P}^{1, \text { an }} \backslash\{0,1, \infty\}$, since its skeleton is a tripod joining the points 0,1 and $\infty$ and $N\left(\mathbb{P}^{1, \text { an }} \backslash\{0,1, \infty\}\right)=\left\{x_{g}\right\}$.

\section{Proof of Theorem C}

Recall that the base field $k$ is assumed to have zero residue characteristic.

10.1. Curves having good reduction. Recall that the skeleton of a smooth projective curve $X$ with good reduction consists of a single point $\eta_{X}$ whose genus equals that of $X$.

Our previous arguments do not apply in the case of smooth projectve curves having good reduction, and we therefore treat this case separately.

Proposition 10.1. Let $k$ be an algebraically closed complete field of zero residue characteristic. Assume that the residue field is countable. Let $X$ be a smooth irreducible projective curve over $k$ with good reduction and of genus at least 2 , and let $U$ be a star-shaped domain. Then the family $\operatorname{Mor}_{k}(U, X)$ is normal.

We shall need for the proof the following weaker version of [CTT16, Lemma 3.6.8]

Lemma 10.2. Let $k$ be an algebraically closed field of zero residue characteristic. Let $U$ be the complement of finitely many closed disks in D. Let $f: U \rightarrow \mathbb{D}$ be an analytic map. Let $I \subset U$ be an interval.

Then there exists a finite subdivision of $I$ into smaller intervals $I_{j}$ such that $\operatorname{diam}(f(z))=a_{j} \operatorname{diam}(z)^{n_{j}}$ for every $z \in I_{j}$.

Proof of Proposition 10.1. Let $f_{n}: U \rightarrow X$ be a sequence of analytic maps. We reduce to the case where the only node $\eta_{U}$ in $U$ is mapped by every $f_{n}$ to the only node $\eta_{X}$ in $X$ by Proposition 8.1 .

The formula (3.1) assures that $g\left(\eta_{X}\right)=g(X) \geq 2$. For every $n \in \mathbb{N}$, the tangent map $d f_{n}\left(\eta_{U}\right): T_{\eta_{U}} U \rightarrow T_{\eta_{X}} X$ is a rational map between the residue curves at $\eta_{U}$ and $\eta_{X}$. By Theorem 9.1 there are only finitely many such nonconstant maps, as the residue curve at $\eta_{X}$ has genus greater than 1 . We may thus assume that all the tangent maps $d f_{n}\left(\eta_{U}\right)$ are equal. Let $d$ be the degree of the $d f_{n}\left(\eta_{U}\right)$.

We treat every connected component of $U \backslash\left\{\eta_{U}\right\}$ separately. Pick any tangent direction $\vec{v}$ at $\eta_{U}$. The image of $U(\vec{v})$ under every $f_{n}$ is contained in some fixed connected component $V$ of $X \backslash\left\{\eta_{X}\right\}$, as all the tangent maps $d f\left(\eta_{U}\right)$ agree. Thus, $f_{n}(U(\vec{v}))$ is contained in some affinoid domain of $X$ for every $n$. Theorem 2.12 implies that there exists a subsequence $f_{n_{j}}$ converging on $U(\vec{v})$ to some continuous map $f_{\infty}$. Since $\tilde{k}$ is countable, we may extract diagonally at every tangent direction at $\eta_{U}$ and obtain a limit map $f_{\infty}: U \rightarrow$ $X$ that is continuous on $U \backslash\left\{\eta_{U}\right\}$.

Observe that $f_{\infty}\left(\eta_{U}\right)=\eta_{X}$. It remains to check that $f_{\infty}$ is continuous at $\eta_{U}$. In order to do so, it suffices to verify that for every sequence of points 
$z_{m} \in U$ converging to $\eta_{U}$ we have $f_{\infty}\left(z_{m}\right) \stackrel{m \rightarrow \infty}{\rightarrow} \eta_{X}$. If the points $z_{m}$ belong to infinitely many different connected components of $U \backslash\left\{\eta_{U}\right\}$, then their images $f_{\infty}\left(z_{m}\right)$ belong to infinitely many distinct connected components of $X \backslash\left\{\eta_{X}\right\}$ and we conclude.

We may thus assume that all the points $z_{m}$ belong to $U(\vec{v})$ for some fixed tangent direction $\vec{v} \in T_{\eta_{U}} U$. Fix an isomorphism $\psi: Y \rightarrow U(\vec{v})$, where $Y$ is an open disk or an open annulus depending on whether $\vec{v}$ is discal or not. Assume that $\psi$ extends continuously to the Gauss point $x_{g}$ with $\lim _{r \rightarrow 1} \varphi\left(\eta_{0, r}\right)=\eta_{U}$. We may assume that for every $m \in \mathbb{N}$ there exists some $0 \leq r<1$ such that $z_{m}=\psi\left(\eta_{0, r}\right)$. Fix an isomorphism $\varphi: \mathbb{D} \rightarrow V$ that extends continuously to $x_{g}$ with $\lim _{r \rightarrow 1} \varphi\left(\eta_{0, r}\right)=\eta_{X}$. Checking the continuity of $f_{\infty}$ at $\eta_{U}$ amounts to showing that $\lim _{r \rightarrow 1} \varphi^{-1} \circ f_{\infty} \circ \psi\left(\eta_{0, r}\right)=$ $x_{g}$.

For every fixed $n \in \mathbb{N} \cup\{\infty\}$, set $F_{n}=\varphi^{-1} \circ f_{n} \circ \psi: Y \rightarrow \mathbb{D}$. The series development $F_{n}(z)=a_{0}^{(n)}+\sum_{i \neq 0} a_{i}^{(n)} z^{i}$ is such that $\left|a_{i}^{(n)}\right| \leq 1$ for all $i \in \mathbb{Z}$, $\left|a_{d}^{(n)}\right|=1$ and $\left|a_{i}^{(n)}\right|<1$ for $i \leq d$, where $d$ denotes the degree of $d f_{n}\left(\eta_{U}\right)$. Consider the segments $I=\left\{\eta_{0, r}: 0 \leq r<1\right\} \subset \mathbb{D}$ and $l_{n}=I \cap F_{n}^{-1}(I)$. Notice that $\operatorname{diam}\left(F_{n}\left(\eta_{0, r_{n}}\right)\right)=\left|a_{0}^{(n)}\right|$, where $r_{n}=\inf \left\{r: \eta_{0, r} \in l_{n}\right\}$.

We distinguish two cases. Assume first that there exists a positive real number $R<1$ such that $\left|a_{0}^{(n)}\right| \leq R$ for infinitely many indices $n \in \mathbb{N}$. After maybe extracting a subsequence, we may reduce $Y$ as to obtain an annulus $Y_{1}$ centered at 0 containing the point $x_{g}$ in its topological boundary and such that every map $F_{n}$ avoids the disk $\overline{\mathbb{D}}(0 ; R)$. Moreover, the skeleton $\mathrm{S}^{\text {an }}\left(Y_{1}\right) \subseteq \bigcap_{n} l_{n}$ is mapped to the segment $I$. For sufficiently large $r$, Lema 10.2 implies that $F_{n}\left(\eta_{0, r}\right)=\eta_{0, r d}$, and so we see that $\lim _{r \rightarrow 1} F_{\infty}\left(\eta_{0, r}\right)=x_{g}$.

Suppose next that $\left|a_{0}^{(n)}\right| \rightarrow 1$. In this case, there exists an open annulus $A \subseteq U(\vec{v})$ whose topological boundary contains $\eta_{U}$ such that the restriction of $f_{\infty}$ to $A$ is the constant map $\eta_{X}$. Indeed, after extracting a subsequence we have that $F_{n}\left(\eta_{0, r_{n}}\right)=\eta_{0, r_{n} d}=\eta_{0,\left|a_{0}^{(n)}\right|}$ by Lemma 10.2. As $\left|a_{0}(n)\right| \rightarrow 1$, then $r_{n} \rightarrow \frac{1}{d}$. Pick any $r>\frac{1}{d}$. For $n \gg 0$, we may assume that $r>r_{n}$. Then, $F_{n}\left(\eta_{0, r}\right)=\eta_{0, r d}$, and since $r d>r_{n} d=\left|a_{0}^{(n)}\right| \rightarrow 1$, we see that $F_{n}\left(\eta_{0, r}\right) \stackrel{n \rightarrow \infty}{\rightarrow} x_{g}$. Thus, $F_{\infty} \equiv x_{g}$ on the open annulus $A\left(\frac{1}{d}, 1\right)$.

10.2. Proof of Theorem C. Assume first that $X$ is a projective curve of genus at least 2 with good reduction. Denote by $\eta_{X}$ its only node. Recall that $X \backslash\left\{\eta_{X}\right\}$ is a countable disjoint union of open disks. Since the open disk and the open annulus have been treated separatedly in Corollary 8.2 , we may assume that $U$ has nodes. The set $\mathrm{N}(U)$ is discrete and consequently we may find a locally finite cover $\left(U_{j}\right)$ of $U$ by basic tubes being either an open disk, an open annulus or a star-shaped domain.

Fix some basic tube $U_{j}$. If the set of nodes of $U_{j}$ is empty, then $U_{j}$ is either a disk or an annulus, and we may apply Corollary 8.2 and Theorem 2.12 to extract a subsequence converging pointwise on $U_{j}$ to some continuous map. 
Suppose now that $U_{j}$ contains one node $\eta_{j}$, i.e. it is a star-shaped domain. Then we apply Proposition 10.1 to extract a subsequence that is pointwise converging on $U_{j}$ to some continuous map. This procedure may be repeated for every open set $U_{j}$, and extracting diagonally we obtain a subsequence $f_{n_{j}}$ that converges on $U$ to some continuous map $f_{\infty}: U \rightarrow X$. This concludes the proof in the case of curves having good reduction.

Suppose now that $X$ is a smooth algebraic curve with negative Euler characteristic whose skeleton is not a single point. Let $\bar{X}$ be the smooth projective curve such that $X$ can be embedded in $\bar{X}$ and $\bar{X} \backslash X$ is a finite set of rigid points.

Let $U$ be any smooth connected boundaryless curve and pick any sequence of analytic maps $f_{n}: U \rightarrow X$. By Theorem $\mathrm{D}$ we may find a locally finite open cover $U_{j}$ of $U$ by basic tubes, and a finite $k$-affinoid cover $\bar{X}_{i}$ of $\bar{X}$ such that for every $n \in \mathbb{N}$ and every $j$ one has $f_{n}\left(U_{j}\right) \subseteq \bar{X}_{i}$ for some $i$. By Theorem 2.12 and a diagonal extraction argument, we may extract a subsequence converging pointwise to some continuous map $f_{\infty}: U \rightarrow \bar{X}$.

By Proposition 3.12, for each index $j$ either we have $f_{\infty}\left(U_{j}\right) \subset X$ or $\left.f_{\infty}\right|_{U_{j}}$ is constant equal to some point in $\bar{X} \backslash X$. If $f_{\infty}(U)$ is not included in $X$, then by continuity and connectedness we conclude that $f_{\infty}$ is constant equal to some point in $\bar{X} \backslash X$ as required.

\section{REFERENCES}

[ACW08] Ta Thi Hoai An, William Cherry, and Julie Tzu-Yueh Wang. Algebraic degeneracy of non-Archimedean analytic maps. Indag. Math. (N.S.), 19(3):481-492, 2008.

[Ber90] Vladimir G. Berkovich. Spectral theory and analytic geometry over nonArchimedean fields, volume 33 of Mathematical Surveys and Monographs. American Mathematical Society, Providence, RI, 1990.

[Ber93] Vladimir G. Berkovich. Étale cohomology for non-Archimedean analytic spaces. Inst. Hautes Études Sci. Publ. Math., (78):5-161 (1994), 1993.

[Ber06] François Berteloot. Méthodes de changement d'échelles en analyse complexe. Annales de la Faculté de Sciences de Toulouse, 15(3):427-483, 2006.

[Bos77] Siegfried Bosch. Eine bemerkenswerte Eigenschaft der formellen Fasern affinoider Räume. Math. Ann., 229(1):25-45, 1977.

[BR10] Matthew Baker and Robert Rumely. Potential theory and dynamics on the Berkovich projective line, volume 159 of Mathematical Surveys and Monographs. American Mathematical Society, Providence, RI, 2010.

[Bro78] Robert Brody. Compact manifolds and hyperbolicity. Trans. Amer. Math. Soc., 235:213-219, 1978.

[Che93] William Cherry. Hyperbolic p-adic analytic spaces. Thesis, Yale University, 1993.

[Che94] William Cherry. Non-Archimedean analytic curves in abelian varieties. Math. Ann., 300(3):393-404, 1994.

[Che96] William Cherry. A non-archimedean analogue of the kobayashi semi-distance and its non-degeneracy on abelian varieties. Illinois Journal of Mathematics, 40(1):123-140, 1996.

[CTT16] Adina Cohen, Michael Temkin, and Dmitri Trushin. Morphisms of Berkovich curves and the different function. Adv. Math., 303:800-858, 2016. 
[DR11] Simone Diverio and Erwan Rousseau. A survey on hyperbolicity of projective hypersurfaces. Publicações Matemáticas do IMPA. [IMPA Mathematical Publications]. Instituto de Matemática Pura e Aplicada (IMPA), Rio de Janeiro, 2011. [On the title page: A survey on hiperbolicity of projective hypersurfaces].

[Duc14] Antoine Ducros. La structure des courbes analytiques. preprint, 2014.

[FKT12] Charles Favre, Jan Kiwi, and Eugenio Trucco. A non-Archimedean Montel's theorem. Compositio Mathematica, 148(3):966-990, 2012.

[Gri15] Nathan Grieve. Diophantine approximation constants for varieties over function fields. preprint, 2015.

[KO75] Shoshichi Kobayashi and Takushiro Ochiai. Meromorphic mappings onto compact complex spaces of general type. Invent. Math., 31(1):7-16, 1975.

[Kob67] Shoshichi Kobayashi. Invariant distances on complex manifolds and holomorphic mappings. J. Math. Soc. Japan, 19:460-480, 1967.

[Kob98] Shoshichi Kobayashi. Hyperbolic complex spaces, volume 318 of Grundlehren der Mathematischen Wissenschaften. Springer-Verlag, Berlin, 1998.

[Lan87] Serge Lang. Introduction to Complex Hyperbolic Spaces. Springer-Verlag, 1987.

[Nog92] Junjiro Noguchi. Meromorphic mappings into compact hyperbolic complex spaces and geometric Diophantine problems. Internat. J. Math., 3(2):277-289, 1992.

[Pet09] Clayton Petsche. Nonarchimedean equidistribution on elliptic curves with global applications. Pacific J. Math., 242(2):345-375, 2009.

[Poi13] Jérôme Poineau. Les espaces de Berkovich sont angéliques. Bulletin de la Société Mathématique de France, 141(2):267-297, 2013.

[Poi14] Jérôme Poineau. Sur les composantes connexes d'une famille d'espaces analytiques p-adiques. Forum Math. Sigma, 2:e14, 21, 2014.

[Roy71] Halsey L. Royden. Remarks on the Kobayashi metric. In Several complex variables, II (Proc. Internat. Conf., Univ. Maryland, College Park, Md., 1970), pages 125-137. Lecture Notes in Math., Vol. 185. Springer, Berlin, 1971.

[RV16] Rita Rodríguez Vázquez. Non-Archimedean normal families. preprint, 2016.

[Sam66] P. Samuel. Lectures on old and new results on algebraic curves. Notes by S. Anantharaman. Tata Institute of Fundamental Research Lectures on Mathematics, No. 36. Tata Institute of Fundamental Research, Bombay, 1966.

[Tem15] Michael Temkin. Introduction to Berkovich analytic spaces. In Berkovich spaces and applications, volume 2119 of Lecture Notes in Math., pages 3-66. Springer, Cham, 2015.

[Thu05] Amaury Thuillier. Potential theory on curves in non-Archimedean geometry. Applications to Arakelov theory. Thesis, Université Rennes 1, October 2005.

[Tsu79] Ryuji Tsushima. Rational maps to varieties of hyperbolic type. Proc. Japan Acad. Ser. A Math. Sci., 55(3):95-100, 1979.

[Voi03] Claire Voisin. On some problems of Kobayashi and Lang; algebraic approaches. In Current developments in mathematics, 2003, pages 53-125. Int. Press, Somerville, MA, 2003.

[Zal75] Lawrence Zalcman. A heuristic principle in complex function theory. Amer. Math. Monthly, 82(8):813-817, 1975.

(Rita Rodríguez Vázquez) CMLS, École Polytechnique, CNRS, Université ParisSaclay, 91128 Palaiseau Cedex, France

E-mail address: rita.rodriguez-vazquez@polytechnique.edu 